\title{
Electroweak sector under scrutiny: A combined analysis of LHC and electroweak precision data
}

\author{
Eduardo da Silva Almeida, ${ }^{1, *}$ Alexandre Alves, ${ }^{2, \dagger}$ N. Rosa-Agostinho, ${ }^{3, \$}$ \\ Oscar J. P. Éboli, ${ }^{1,}$ and M. C. Gonzalez-Garcia ${ }^{3,4,5, \|}$ \\ ${ }^{1}$ Instituto de Física, Universidade de São Paulo, São Paulo 05508-090, Brazil \\ ${ }^{2}$ Departamento de Física, Universidade Federal de São Paulo, \\ UNIFESP, Diadema, São Paulo 09972-270, Brazil \\ ${ }^{3}$ Departament de Fisica Quantica i Astrofisica and Institut de Ciencies del Cosmos, \\ Universitat de Barcelona, Diagonal 647, E-08028 Barcelona, Spain \\ ${ }^{4}$ Institució Catalana de Recerca i Estudis Avancats (ICREA), \\ Pg. Lluis Companys 23, 08010 Barcelona, Spain \\ ${ }^{5}$ C.N. Yang Institute for Theoretical Physics, Stony Brook University, \\ Stony Brook, New York 11794-3849, USA
}

(Received 11 December 2018; published 6 February 2019)

\begin{abstract}
We perform a comprehensive study of the Higgs couplings, gauge boson couplings to fermions, and triple gauge boson vertices. We work in the framework of effective theories including the effects of the dimension-six operators contributing to these observables. We determine the presently allowed range for the coefficients of these operators via a 20 parameter global fit to the electroweak precision data, as well as electroweak diboson and Higgs production data from LHC runs 1 and 2. We quantify the improvement on the determination of the 20 Wilson coefficients by the inclusion of the run 2 results. In particular, we present a novel analysis of the ATLAS run $236.1 \mathrm{fb}^{-1}$ data on the transverse mass distribution of $W^{+} W^{-}$ and $W^{ \pm} Z$ in the leptonic channel, which allows for stronger tests of the triple gauge boson vertices. We discuss the discrete (quasi)degeneracies existing in the parameter space of operator coefficients relevant for the Higgs couplings to fermions and gauge bosons. In particular, we show how the inclusion of the incipient $t H$ data can break those degeneracies in the determination of the top Higgs coupling. We also discuss and quantify the effect of keeping the terms quadratic in the Wilson coefficients in the analysis, and we show the importance of the Higgs data to constrain some of the operators that modify the triple gauge boson couplings in the linear regime.
\end{abstract}

DOI: $10.1103 /$ PhysRevD.99.033001

\section{INTRODUCTION}

Since the discovery of a new state resembling the SM Higgs boson [1,2], the CERN Large Hadron Collider (LHC) has accumulated an impressive amount of data that allow new searches for extensions of the Standard Model (SM), as well as detailed studies of the SM predictions. Presently, the negative results of the search for direct new physics effects indicate that new states are probably heavy; therefore, there might exist a mass gap between the SM

\footnotetext{
*eduardo.silva.almeida@usp.br

†aalves.unifesp@gmail.com

"nuno.f.agostinho@gmail.com

§oeboli@gmail.com

"maria.gonzalez-garcia@stonybrook.edu
}

Published by the American Physical Society under the terms of the Creative Commons Attribution 4.0 International license. Further distribution of this work must maintain attribution to the author(s) and the published article's title, journal citation, and DOI. Funded by SCOAP ${ }^{3}$. states and the new ones. In this scenario, hints on the new physics can manifest itself first through deviations from the SM predictions.

Against this background, effective Lagrangians appear to be a most adequate tool for scientific advancement. The effective Lagrangian approach [3-5] is a modelindependent framework, which, using as inputs the low energy particle contents and symmetries, describes new physics that is expected to manifest itself directly at an energy scale $\Lambda$ larger than the scale at which the experiments are performed, by including in the Lagrangian higherdimension operators. In this context, and within the present experimental results, we proceed by assuming that the observed scalar belongs indeed to a light electroweak doublet and that the $S U(2)_{L} \otimes U(1)_{Y}$ symmetry is linearly realized in the effective theory. Barring effects associated with violation of total lepton number, the lowest-order operators which can be built are of dimension six. The coefficients of these dimension-six operators parametrize our ignorance of the new physics effects, and our task at hand is to determine them using the available data. 
In this work, we follow this road by performing a comprehensive analysis of the observables related to the electroweak sector, which at present allows for precision tests of the couplings between electroweak gauge bosons and fermions, triple electroweak gauge couplings (TGC), and the couplings of the Higgs to fermions and gauge bosons. The first two sets of couplings allow us to probe the $S U(2)_{L} \otimes U(1)_{Y}$ gauge structure of the SM, whereas the last one aims to study the couplings of the recently discovered scalar, i.e., to directly probe the electroweak symmetry-breaking mechanism. In this respect, this work extends and updates our previous partial constrains on Higgs anomalous couplings and TGCs [6-9].

In the framework of the effective Lagrangian described above (also recently labeled in the literature as the SMEFT), our study involves 20 dimension-six operators whose coefficients are determined by means of a global fit to the relevant data, including low-energy electroweak precision measurements as well as LHC data on gauge boson pair production and Higgs observables. The global analysis approach (see Refs. [10,11] for recent related works) is becoming mandatory because, within the present LHC statistics, changes in the couplings of gauge bosons to fermions, even within the constraints of electroweak precision data (EWPD), can lead to modifications of the kinematical distributions in gauge boson pair production at LHC of comparable size to the ones stemming from the purely anomalous TGC [12-14]. These, in turn, influence the determination of the Wilson coefficients for the operators entering the Higgs observables.

With this aim, we briefly introduce in Sec. II the set of dimension-six operators included in our study and point out the main sources of (quasi)degenerate solutions which appear in the analysis. Section III contains a brief description of the data and statistical treatment applied to it. For the Higgs observables, this includes the final results of the LHC run 1 and the most up to date from run 2 in terms of signal strengths or ratios of cross sections and branching ratios. Concerning the gauge boson pair production data, besides the final results of the LHC run 1, we perform a novel analysis of the ATLAS data on transverse mass distribution of $W^{+} W^{-}$and $W^{ \pm} Z$ in the leptonic channel. The body of our results is discussed in Sec. IV, which we present in terms of the different sectors tested: gauge boson fermion couplings, TGCs, and Higgs couplings. One particularly interesting observation is the relevance of the incipient $t H$ data to break possible degeneracies in the determination of the top Yukawa coupling. We finish Sec. IV with a quantification of the relevance of keeping the terms quadratic in the Wilson coefficients in the analysis. In that respect, our results show that the accumulated statistics on the Higgs observables is starting to be large enough for meaningful constraints on the Wilson coefficients of some of the operators to be imposed from their interference with the SM contributions. We summarize our findings in Sec. V.

\section{THEORETICAL FRAMEWORK}

Up to now, there is no direct evidence of new states being produced at the LHC. Therefore, we parametrize possible deviations from the SM by higher-dimension operators:

$$
\mathcal{L}_{\text {eff }}=\mathcal{L}_{\mathrm{SM}}+\sum_{n>4, j} \frac{f_{n, j}}{\Lambda^{n-4}} \mathcal{O}_{n, j},
$$

where the $\mathrm{SM} S U(3)_{C} \otimes S U(2)_{L} \otimes U(1)_{Y}$ gauge symmetry is realized linearly in the $\mathcal{O}_{n, j}$ operators. For the sake of simplicity, we retain only the dimension-six operators that conserve $C, P$, and lepton and baryon numbers. The first higher-order operator is of dimension five [15]; however, it does not contribute to the LHC physics due to the strong constraints originating from the neutrino sector. The first operators to play a significant role at the LHC are of dimension six, i.e., $n=6$. It is well known that there are 59 independent dimension-six operators [16], up to flavor and hermitian conjugation. Using the freedom in the choice of basis [17-20] due to the use of equations of motion (EOM), we work in that of Hagiwara, Ishihara, Szalapski, and Zeppenfeld (HISZ) [21,22] for the pure bosonic operators.

In what follows, we focus on the subset of the dimensionsix operator basis that impacts the precision electroweak data (EWPD) [23], TGCs, and Higgs physics. The EWPD observables receive linear contributions from seven operators involving fermions, gauge bosons, and the Higgs field:

$$
\begin{aligned}
& \mathcal{O}_{\Phi L, i j}^{(1)}=\Phi^{\dagger}\left(i \stackrel{\leftrightarrow}{D_{\mu}} \Phi\right)\left(\bar{L}_{i} \gamma^{\mu} L_{j}\right), \\
& \mathcal{O}_{\Phi L, i j}^{(3)}=\Phi^{\dagger}\left(i \stackrel{\leftrightarrow}{D}{ }_{\mu}{ }_{\mu} \Phi\right)\left(\bar{L}_{i} \gamma^{\mu} T_{a} L_{j}\right), \\
& \mathcal{O}_{\Phi Q, i j}^{(1)}=\Phi^{\dagger}\left(i \stackrel{\leftrightarrow}{D_{\mu}} \Phi\right)\left(\bar{Q}_{i} \gamma^{\mu} Q_{j}\right), \\
& \mathcal{O}_{\Phi Q, i j}^{(3)}=\Phi^{\dagger}\left(i \stackrel{\leftrightarrow}{D}{ }_{\mu} \Phi\right)\left(\bar{Q}_{i} \gamma^{\mu} T_{a} Q_{j}\right), \\
& \mathcal{O}_{\Phi u, i j}^{(1)}=\Phi^{\dagger}\left(i \stackrel{\leftrightarrow}{D}_{\mu} \Phi\right)\left(\bar{u}_{R_{i}} \gamma^{\mu} u_{R_{j}}\right), \\
& \mathcal{O}_{\Phi d, i j}^{(1)}=\Phi^{\dagger}\left(i \stackrel{\leftrightarrow}{D_{\mu}} \Phi\right)\left(\bar{d}_{R_{i}} \gamma^{\mu} d_{R_{j}}\right), \\
& \mathcal{O}_{\Phi e, i j}^{(1)}=\Phi^{\dagger}\left(i \stackrel{\leftrightarrow}{D}_{\mu} \Phi\right)\left(\bar{e}_{R_{i}} \gamma^{\mu} e_{R_{j}}\right) \text {, }
\end{aligned}
$$

together with a purely four fermion operator:

$$
\mathcal{O}_{L L L L}=\left(\bar{L} \gamma^{\mu} L\right)\left(\bar{L} \gamma^{\mu} L\right)
$$

In addition to the above fermionic operators, there are two bosonic operators that contribute to the EWPD,

$$
\begin{aligned}
\mathcal{O}_{B W} & =\Phi^{\dagger} \hat{B}_{\mu \nu} \hat{W}^{\mu \nu} \Phi \\
\text { and } \quad \mathcal{O}_{\Phi, 1} & =\left(D_{\mu} \Phi\right)^{\dagger} \Phi \Phi^{\dagger}\left(D^{\mu} \Phi\right) .
\end{aligned}
$$


In the equations above, $\Phi$ stands for the SM Higgs doublet, whereas the lepton (quark) doublet is denoted by $L(Q)$, and $f_{R}$ is the $S U(2)_{L}$ singlet fermions, where $i, j$ are family indices. In addition, we defined $\Phi^{\dagger} \stackrel{\leftrightarrow}{D}_{\mu} \Phi=\Phi^{\dagger} D_{\mu} \Phi-$ $\left(D_{\mu} \Phi\right)^{\dagger} \Phi \quad$ and $\quad \Phi^{\dagger} \stackrel{\leftrightarrow}{D^{a}}{ }_{\mu} \Phi=\Phi^{\dagger} T^{a} D_{\mu} \Phi-\left(D_{\mu} \Phi\right)^{\dagger} T^{a} \Phi$ with $T^{a}=\sigma^{a} / 2$. Also, we defined $\hat{B}_{\mu \nu} \equiv i\left(g^{\prime} / 2\right) B_{\mu \nu}$ and $\hat{W}_{\mu \nu} \equiv i(g / 2) \sigma^{a} W_{\mu \nu}^{a}$, with $g$ and $g^{\prime}$ being the $S U(2)_{L}$ and $U(1)_{Y}$ gauge couplings, respectively. The Pauli matrices are denoted by $\sigma^{a}$.

The seven operators in Eq. (2.2) modify directly the coupling of the $Z$ to fermion pairs, whereas $\mathcal{O}_{\Phi Q, i j}^{(3)}$ contributes also to the $W$ couplings to left quarks. $\mathcal{O}_{L L L L}, \mathcal{O}_{B W}$, and $\mathcal{O}_{\Phi, 1}$ contributions are ubiquitous and stem from their effect on the finite renormalization of the SM fields and couplings once the Lagrangian is canonically normalized. In particular, $\mathcal{O}_{L L L L}$ gives a finite contribution to the Fermi constant, whereas the operators $\mathcal{O}_{B W}$ and $\mathcal{O}_{\Phi, 1}$ lead to finite correction to the $S$ and $T$ oblique parameters, respectively. Furthermore, in the analysis of EWPD, we did not considered six dipole operators whose interference with the SM contributions vanish for EWPD observables.

In what follows, in order to avoid the existence of blind directions $[24,25]$ in the EWPD analyses, we used the freedom associated with the use of EOM to remove the operator combinations,

$$
\sum_{i} \mathcal{O}_{\Phi L, i i}^{(1)} \text { and } \sum_{i} \mathcal{O}_{\Phi L, i i}^{(3)}
$$

from our operator basis. Moreover, we assume no family mixing in the above operators to prevent the generation of too large flavor violation, and for simplicity, we consider the operators to be generation independent; hereafter, we drop the generation indexes for these operators. With these hypotheses, the operators $\mathcal{O}_{\Phi L}^{(1)}$ and $\mathcal{O}_{\Phi L}^{(3)}$ are removed by the use of EOM [7].

With the above assumptions, in our basis, only the operator $\mathcal{O}_{\Phi e}^{(1)}$ modifies the $Z$ coupling to leptons, whereas there are additional contributions to the $Z$-quark pair vertices originating from $\mathcal{O}_{\Phi u}^{(1)}, \mathcal{O}_{\Phi d}^{(1)}, \mathcal{O}_{\Phi Q}^{(1)}$, and $\mathcal{O}_{\Phi Q}^{(3)}$. Moreover, the $W$ coupling to fermions receives extra contributions from $\mathcal{O}_{\Phi Q}^{(3)}$; see Table I.

Altogether the part of the dimension-six effective Lagrangian that contributes to the EWPD is

$$
\begin{aligned}
\Delta \mathcal{L}_{\mathrm{eff}}^{\mathrm{EWPD}}= & \frac{f_{\Phi Q}^{(1)}}{\Lambda^{2}} \mathcal{O}_{\Phi Q}^{(1)}+\frac{f_{\Phi Q}^{(3)}}{\Lambda^{2}} \mathcal{O}_{\Phi Q}^{(3)}+\frac{f_{\Phi u}^{(1)}}{\Lambda^{2}} \mathcal{O}_{\Phi u}^{(1)} \\
& +\frac{f_{\Phi d}^{(1)}}{\Lambda^{2}} \mathcal{O}_{\Phi d}^{(1)}+\frac{f_{\Phi e}^{(1)}}{\Lambda^{2}} \mathcal{O}_{\Phi e}^{(1)}+\frac{f_{B W}}{\Lambda^{2}} \mathcal{O}_{B W} \\
& +\frac{f_{\Phi, 1}}{\Lambda^{2}} \mathcal{O}_{\Phi, 1}+\frac{f_{L L L L}}{\Lambda^{2}} \mathcal{O}_{L L L L}
\end{aligned}
$$

TABLE I. Anomalous couplings to fermions generated by the dimension-six operators considered in the analysis.

\begin{tabular}{lccccc}
\hline \hline & $H \bar{f} f$ & $Z \bar{q} q$ & $Z \bar{l} l$ & $W \bar{u} d$ & $W \bar{l} \nu$ \\
\hline $\mathcal{O}_{B W}$ & & $\mathrm{X}$ & $\mathrm{X}$ & $\mathrm{X}$ & $\mathrm{X}$ \\
$\mathcal{O}_{\Phi, 1}$ & $\mathrm{X}$ & $\mathrm{X}$ & $\mathrm{X}$ & $\mathrm{X}$ & $\mathrm{X}$ \\
$\mathcal{O}_{\Phi, 2}$ & $\mathrm{X}$ & $\mathrm{X}$ & & & \\
$\mathcal{O}_{\Phi Q}^{(1)}, \mathcal{O}_{\Phi u}^{(1)}, \mathcal{O}_{\Phi d}^{(1)}$ & & $\mathrm{X}$ & & $\mathrm{X}$ & \\
$\mathcal{O}_{\Phi Q}^{(1)}$, & & & $\mathrm{X}$ & & \\
$\mathcal{O}_{\Phi e}^{(1)}$, & & & & $\mathrm{X}$ & \\
$\mathcal{O}_{\Phi u d}^{(1)}$ & & & & & \\
$\mathcal{O}_{u \Phi, 33}^{(1)}$ & $\mathrm{X}($ if $f=t)$ & & & & \\
$\mathcal{O}_{d \Phi, 33}$ & $\mathrm{X}$ (if $f=b)$ & & & & \\
$\mathcal{O}_{e \Phi, 33}$ & $\mathrm{X}($ if $f=\tau)$ & & & & \\
$\mathcal{O}_{u \Phi, 22}$ & $\mathrm{X}$ (if $f=\mu)$ & & & & \\
\hline \hline
\end{tabular}

As is well known, the above operators are strongly constrained by the EWPD observables [26].

Data on electroweak diboson production processes (here on EWDBD) at the LHC, $p p \rightarrow W^{+} W^{-}$, and $p p \rightarrow Z W^{ \pm}$, can be used to study operators that change the $W$ and $Z$ couplings to fermions, as well as TGC. These processes receive contributions from the previously discussed operators, as well as from the fermionic operator,

$$
\mathcal{O}_{\Phi u d}^{(1)}=\tilde{\Phi}^{\dagger}\left(i \stackrel{\leftrightarrow}{D}_{\mu} \Phi\right)\left(\bar{u}_{R} \gamma^{\mu} d_{R}+\text { H.c. }\right)
$$

that modifies the couplings of $W$ to right-handed quark pairs and does not interfere with the SM contributions to the EWPD observables at the order considered in the analysis. In addition, TGCs are also modified by two additional dimension-six operators that include Higgs and electroweak gauge fields in the HISZ basis,

$$
\begin{aligned}
\mathcal{O}_{W} & =\left(D_{\mu} \Phi\right)^{\dagger} \hat{W}^{\mu \nu}\left(D_{\nu} \Phi\right) \\
\text { and } \quad \mathcal{O}_{B} & =\left(D_{\mu} \Phi\right)^{\dagger} \hat{B}^{\mu \nu}\left(D_{\nu} \Phi\right),
\end{aligned}
$$

and by one operator that contains exclusively gauge bosons,

$$
\mathcal{O}_{W W W}=\operatorname{Tr}\left[\hat{W}_{\mu}^{\nu} \hat{W}_{\nu}^{\rho} \hat{W}_{\rho}^{\mu}\right]
$$

We present in Table II the anomalous TGC stemming from the dimension-six operators in our basis. It is interesting to notice that dimension-six operators do not give rise to anomalous TGC among neutral gauge bosons. We define the effective Lagrangian of the operators that contribute to TGC in addition to the ones participating in the EWPD analysis as 
$\Delta \mathcal{L}_{\text {eff }}^{\mathrm{TGC}}=\frac{f_{W W W}}{\Lambda^{2}} \mathcal{O}_{W W W}+\frac{f_{W}}{\Lambda^{2}} \mathcal{O}_{W}+\frac{f_{B}}{\Lambda^{2}} \mathcal{O}_{B}+\frac{f_{\Phi u d}^{(1)}}{\Lambda^{2}} \mathcal{O}_{\Phi u d}^{(1)}$.

Concerning Higgs processes, in order to quantify possible deviations from the SM predictions, we must consider some additional dimension-six operators that contain the Higgs field $\Phi$. In particular, the following fermionic operators modify the Yukawa couplings of the Higgs boson:

$$
\begin{aligned}
\mathcal{O}_{e \Phi, i j} & =\left(\Phi^{\dagger} \Phi\right)\left(\bar{L}_{i} \Phi e_{R, j}\right), \\
\mathcal{O}_{u \Phi, i j} & =\left(\Phi^{\dagger} \Phi\right)\left(\bar{Q}_{i} \tilde{\Phi} u_{R, j}\right), \\
\text { and } \quad \mathcal{O}_{d \Phi, i j} & =\left(\Phi^{\dagger} \Phi\right)\left(\bar{Q}_{i} \Phi d_{R, j}\right),
\end{aligned}
$$

with $\tilde{\Phi}=i \sigma_{2} \Phi^{*}$ and $i, j$ being generation indices. In this work, we consider only the diagonal couplings of Higgs to the third family and also to muon pairs (which are the only ones being currently tested at LHC); that is, we work with the effective Lagrangian:

$$
\begin{aligned}
\Delta \mathcal{L}_{\text {eff }}^{\text {Yuk }}= & \frac{f_{\mu} m_{\mu}}{\Lambda^{2} v} \mathcal{O}_{e \Phi, 22}+\frac{f_{\tau} m_{\tau}}{\Lambda^{2} v} \mathcal{O}_{e \Phi, 33}+\frac{f_{b} m_{b}}{\Lambda^{2} v} \mathcal{O}_{d \Phi, 33} \\
& +\frac{f_{t} m_{t}}{\Lambda^{2} v} \mathcal{O}_{u \Phi, 33}+\text { H.c. }
\end{aligned}
$$

Finally, the couplings of the Higgs to gauge bosons are further modified by the bosonic operators,

$$
\begin{array}{ll}
\mathcal{O}_{G G}=\Phi^{\dagger} \Phi G_{\mu \nu}^{a} G^{a \mu \nu}, & \mathcal{O}_{W W}=\Phi^{\dagger} \hat{W}_{\mu \nu} \hat{W}^{\mu \nu} \Phi, \\
\mathcal{O}_{B B}=\Phi^{\dagger} \hat{B}_{\mu \nu} \hat{B}^{\mu \nu} \Phi, & \mathcal{O}_{\Phi, 2}=\frac{1}{2} \partial^{\mu}\left(\Phi^{\dagger} \Phi\right) \partial_{\mu}\left(\Phi^{\dagger} \Phi\right) .
\end{array}
$$

The operator $\mathcal{O}_{\Phi, 2}$ modifies all Higgs couplings due to a finite renormalization of the Higgs wave function. We keep its effects explicitly in the modification of the Higgs vertices generated from the dimension-four part of the Lagrangian, $\mathcal{L}_{\mathrm{SM}}$. Corrections to vertices generated by other dimension-six operators are absorbed in the redefinition of the corresponding Wilson coefficient.

As for the effective couplings to gluons, in what follows, we work in the convention that the Wilson coefficient of the operator $\mathcal{O}_{G G}$ contains not only contributions of new possible colored states appearing in the loop connecting gluon pairs to the Higgs, but also the effects of the anomalous operators that modify the SM Yukawa coupling to bottom and top quarks running in the loop. The effective Lagrangian associated to these operators is
TABLE II. Anomalous couplings of gauge and Higgs bosons induced the dimension-six operators the we consider.

\begin{tabular}{lcccccc}
\hline \hline & $Z W W$ & $\gamma W W$ & $H \gamma \gamma$ & $H Z Z$ & $H Z \gamma$ & $H W W$ \\
\hline $\mathcal{O}_{W W W}$ & $\mathrm{X}$ & $\mathrm{X}$ & & & & \\
$\mathcal{O}_{W}$ & $\mathrm{X}$ & $\mathrm{X}$ & & $\mathrm{X}$ & $\mathrm{X}$ & $\mathrm{X}$ \\
$\mathcal{O}_{B}$ & $\mathrm{X}$ & $\mathrm{X}$ & & $\mathrm{X}$ & $\mathrm{X}$ & \\
$\mathcal{O}_{B W}$ & $\mathrm{X}$ & $\mathrm{X}$ & $\mathrm{X}$ & $\mathrm{X}$ & $\mathrm{X}$ & $\mathrm{X}$ \\
$\mathcal{O}_{W W}$ & & & $\mathrm{X}$ & $\mathrm{X}$ & $\mathrm{X}$ & $\mathrm{X}$ \\
$\mathcal{O}_{B B}$ & & & $\mathrm{X}$ & $\mathrm{X}$ & $\mathrm{X}$ & \\
$\mathcal{O}_{\Phi, 1}$ & $\mathrm{X}$ & & & $\mathrm{X}$ & & $\mathrm{X}$ \\
$\mathcal{O}_{\Phi, 2}$ & & & & $\mathrm{X}$ & & $\mathrm{X}$ \\
\hline \hline
\end{tabular}

$$
\begin{aligned}
\Delta \mathcal{L}_{\mathrm{eff}}^{\mathrm{HVV}}= & -\frac{\alpha_{s}}{8 \pi} \frac{f_{G G}}{\Lambda^{2}} \mathcal{O}_{G G}+\frac{f_{B B}}{\Lambda^{2}} \mathcal{O}_{B B} \\
& +\frac{f_{W W}}{\Lambda^{2}} \mathcal{O}_{W W}+\frac{f_{\Phi, 2}}{\Lambda^{2}} \mathcal{O}_{\Phi, 2} .
\end{aligned}
$$

Notice that we rescale the Wilson coefficient $f_{G G} / \Lambda^{2}$ of the gluon-gluon operator to include a loop suppression factor such that its limits are of the same order of the Wilson coefficients of other operators.

In summary, the total effective Lagrangian that we consider is

$$
\begin{aligned}
\mathcal{L}_{\text {eff }}= & \mathcal{L}_{\mathrm{SM}}+\Delta \mathcal{L}_{\text {eff }}^{\mathrm{EWPD}}+\Delta \mathcal{L}_{\text {eff }}^{\mathrm{TGC}}+\Delta \mathcal{L}_{\text {eff }}^{\mathrm{Yuk}} \\
& +\Delta \mathcal{L}_{\text {eff }}^{\mathrm{HVV}} .
\end{aligned}
$$

Tables I and II show the anomalous three point vertices generated by the effective Lagrangian in Eq. (2.15); the explicit form of the couplings and the different Lorentz structures generated can be found, for example, in Refs. $[7,14,26,27]$ to which we refer the reader for details.

In brief, EWPD constrains at tree level only the eight Wilson coefficients of the operators appearing in $\Delta \mathcal{L}_{\text {eff }}^{\text {EWPD }}$, whereas the TGC analysis of EWDBD receives contributions from the 12 operators in $\Delta \mathcal{L}_{\text {eff }}^{\mathrm{EWPD}}$ and $\Delta \mathcal{L}_{\text {eff }}^{\mathrm{TGC}}$. On the other hand, 19 of the 20 operators in $\mathcal{L}_{\text {eff }}$ are required to study the different Higgs production and decay channels at LHC; in fact, only $\mathcal{O}_{W W W}$ does not contribute to Higgs processes. Altogether, at dimension six, the global analysis of EWPD, EWDBD, and Higgs processes involves the 20 operators in Eq. (2.15).

As we will see in the following section, at present, there is enough experimental information to individually bound the 20 Wilson coefficients, but there are still important discrete (quasi)degeneracies. They can be understood in terms of sign flips of the couplings of the Higgs to gauge bosons and to fermions with respect to the SM. For instance, the coefficient of the $H W_{\mu}^{+} W^{-\mu}$ vertex is

$$
\left(\frac{g^{2} v}{2}\right)\left[1-\frac{v^{2}}{4}\left(\frac{f_{\Phi, 1}}{\Lambda^{2}}+2 \frac{f_{\Phi, 2}}{\Lambda^{2}}\right)\right] .
$$


As $f_{\Phi, 1} / \Lambda^{2}$ possesses a stringent bound from EWPD, we anticipate a degeneracy with the SM results for $f_{\Phi, 2} / \Lambda^{2}=0$ and around $f_{\Phi, 2} / \Lambda^{2}=4 / v^{2} \sim 65 \mathrm{TeV}^{-2}$. These points in parameter space are also nearly degenerate for the vertex $H Z_{\mu} Z^{\mu}$.

For the Higgs couplings to fermions, anomalous interactions can also lead to Yukawa couplings on the order of the SM ones but with a different sign as the coefficient of the $H \bar{f} f$ vertex is now

$$
-\frac{m_{f}}{v}\left[1-\frac{v^{2}}{2}\left(\frac{f_{\Phi, 2}}{\Lambda^{2}}+\sqrt{2} \frac{f_{f}}{\Lambda^{2}}\right)\right],
$$

where $f=\mu, \tau, b, t$. As $f_{\Phi, 2} / \Lambda^{2}$ has two different values compatible with flipping the sign of the SM $H V V$ coupling, we can anticipate that $f_{f} / \Lambda^{2}$ will have $2 \times 2$ degenerate SM-like solutions, two corresponding to $f_{f} / \Lambda=0$, and the other two with $f_{f} / \Lambda= \pm 2 \sqrt{2} / v^{2} \sim 45 \mathrm{TeV}^{-2}$.

A further source of degeneracy is the effective gluongluon-Higgs interaction associated to the operator $H G_{\mu \nu}^{a} G^{a, \mu \nu}$ whose coefficient is

$$
-\frac{1}{4} G_{\mathrm{SM}}^{g g}-\frac{\alpha_{S} v}{8 \pi} \frac{f_{G G}}{\Lambda^{2}},
$$

where $G_{\mathrm{SM}}^{g g} \sim-5.3 \times 10^{-2} \mathrm{TeV}^{-1}$ summarizes the $\mathrm{SM}$ one-loop contribution. Flipping the sign of the SM contribution leads to the existence of a SM-like solution for $f_{G G} / \Lambda^{2} \sim-4 \pi /\left(v \alpha_{s}\right) G_{g g, \mathrm{SM}} \sim 25 \mathrm{TeV}^{-2}$. The equivalent effect is present in the photon-photon-Higgs coupling $H F_{\mu \nu} F^{\mu \nu}$ with a coefficient

$$
-\frac{1}{4} G_{\mathrm{SM}}^{\gamma \gamma}+\frac{e^{2} v}{4} \frac{f_{W W}+f_{B B}-f_{B W}}{\Lambda^{2}},
$$

where $G_{\mathrm{SM}}^{\gamma \gamma} \sim 3.3 \times 10^{-2} \mathrm{TeV}^{-1}$, and a SM-like solutions for the $H \gamma \gamma$ decay can be found for $\left(f_{W W}+f_{B B}-f_{B W}\right) /$ $\Lambda^{2} \sim 2 /\left(v e^{2}\right) G_{\gamma \gamma, \mathrm{SM}} \sim 3 \mathrm{TeV}^{-2}$. This degeneracy, however, is only approximate because EWPD independently constrains $f_{B W}$, and the measurement of the effective photonZ-coupling $H F_{\mu \nu} Z^{\mu \nu}$ bounds a different combination of $f_{W W}, f_{B B}$, and $f_{B W}$.

\section{ANALYSES FRAMEWORK}

In order to constrain the Wilson coefficients of the dimension-six operators in the effective Lagrangian Eq. (2.15), we considered the EWPD, diboson production, and Higgs signal strengths. In the EWPD analysis, we take into account 15 observables, of which 12 are $Z$ observables [23]:
$\Gamma_{Z}, \sigma_{h}^{0}, \mathcal{A}_{\ell}\left(\tau^{\mathrm{pol}}\right), R_{\ell}^{0}, \mathcal{A}_{\ell}(\mathrm{SLD}), A_{\mathrm{FB}}^{0, l}, R_{c}^{0}, R_{b}^{0}, \mathcal{A}_{c}, \mathcal{A}_{b}, A_{\mathrm{FB}}^{0, c}$, and $A_{\mathrm{FB}}^{0, b}(\mathrm{SLD} / \mathrm{LEP}-\mathrm{I})$,

supplemented by three $W$ observables,

$$
M_{W}, \Gamma_{W} \text { and } \operatorname{Br}(W \rightarrow \ell \nu)
$$

that are, respectively, its average mass taken from [28], its width from LEP2/Tevatron [29], and the leptonic $W$ branching ratio for which the average in Ref. [28] is considered. In order to perform the statistical analysis, we constructed a $\chi^{2}$ function for the EWPD,

$\chi_{\mathrm{EWPD}}^{2}\left(f_{B W}, f_{\Phi, 1}, f_{\Phi, Q}^{(3)}, f_{\Phi, Q}^{(1)}, f_{\Phi, u}^{(1)}, f_{\Phi, d}^{(1)}, f_{\Phi, e}^{(1)}, f_{L L L L}\right)$.

We include in our EWPD analysis the correlations among the above observables as displayed in Ref. [23]. Furthermore, the SM predictions and their uncertainties due to variations of the SM parameters were extracted from [30]. For further details of this part of the statistical analysis, we refer the reader to Refs. [14,26].

The structure of the electroweak triple gauge boson coupling has been the subject of direct scrutiny in gauge boson pair production at LEP2 [31] and the run 1 of LHC, where the ATLAS and CMS collaborations have used their full data samples on $W^{+} W^{-}[32,33]$ and $W^{ \pm} Z[34,35]$ productions to constrain the possible deviations of TGCs from the SM structure in terms of the effective Lorentz invariant parametrization of Ref. [36] or in terms of coefficients of some of the relevant dimension-six operators. For run 2, the number of experimental studies aiming at deriving the corresponding limits is still rather sparse. In particular, ATLAS [37] has presented some results on bounds on TGC couplings from $W Z$ production but still with data collected with $13.3 \mathrm{fb}^{-1}$. With this limited luminosity, the TGC sensitivity is still below that of run 1 . However, ATLAS has also presented results on their measurements of diboson production at $13 \mathrm{TeV}$ with higher luminosity, and these data can be used to set better constraints on TGC. With this aim, here, we use the ATLAS results on $W Z$ production [38] and on $W W$ [39] both with $36.1 \mathrm{fb}^{-1}$ as we describe next.

In order to obtain the bounds on the Wilson coefficients in the effective Lagrangian in Eq. (2.15), we study the $W^{+} W^{-}$and $W^{ \pm} Z$ productions in the leptonic channel using the available kinematic distribution that is most sensitive for TGC analysis. More specifically, the channels that we analyze and their kinematical distributions are: 


\begin{tabular}{lcccc}
\hline Channel $(a)$ & Distribution & \# bins & Data set & Int Lum \\
\hline$W W \rightarrow \ell^{+} \ell^{\prime-}+E_{T}(0 j)$ & $p_{T}^{\text {leading,lepton }}$ & 3 & ATLAS 8 TeV, & $20.3 \mathrm{fb}^{-1}[32]$ \\
$W W \rightarrow \ell^{+} \ell^{(\prime)}+E_{T}(0 j)$ & $m_{\ell \ell^{(\prime)}}$ & 8 & CMS 8 TeV, & $19.4 \mathrm{fb}^{-1}[33]$ \\
$W Z \rightarrow \ell^{+} \ell^{-} \ell^{(\prime) \pm}$ & $m_{T}^{W Z}$ & 6 & ATLAS 8 TeV, & $20.3 \mathrm{fb}^{-1}[34]$ \\
$W Z \rightarrow \ell^{+} \ell^{-} \ell^{(\prime) \pm}+E_{T}$ & $Z$ candidate $p_{T}^{\ell \ell}$ & 10 & CMS 8 TeV, & $19.6 \mathrm{fb}^{-1}[35]$ \\
$W W \rightarrow e^{ \pm} \mu^{\mp}+E_{T}(0 j)$ & $m_{T}$ & 17 & ATLAS 13 TeV, & $36.1 \mathrm{fb}^{-1}[39]$ \\
$W Z \rightarrow \ell^{+} \ell^{-} \ell^{(\prime) \pm}$ & $m_{T}^{W Z}$ & 6 & ATLAS 13 TeV, & $36.1 \mathrm{fb}^{-1}[38]$ \\
\hline
\end{tabular}

For each experiment and channel, we extract from the experimental publications the observed event rates in each bin, $N_{i, \mathrm{~d}}^{a}$, as well as the background expectations $N_{i, \mathrm{bck}}^{a}$, and the SM $W^{+} W^{-}\left(W^{ \pm} Z\right)$ predictions, $N_{i, \mathrm{sm}}^{a}$.

For details of the analysis of EWDBD from run 1, we refer the reader to Ref. [14] that contains our procedure as well as its validation against the TGC results from the experimental collaborations.

Concerning the run 2 EWDBD analysis, for the $W^{+} W^{-}$ final state, we study the transverse mass distribution in the ATLAS $13 \mathrm{TeV}$ ggF sample. We extract from Fig. 4 in Ref. [39] the data, the non-WW backgrounds, as well as the SM $W W$ contributions in each of the 17 bins in the transverse mass variable,

$$
m_{T} \equiv \sqrt{\left(E_{T}^{\ell \ell}+E_{T}^{\text {miss }}\right)^{2}-\left|\vec{p}_{T}^{\ell \ell}+\vec{p}_{T}^{\text {miss }}\right|^{2}}
$$

with $E_{T}^{\ell \ell}=\sqrt{\left|\vec{p}_{T}^{\ell \ell}\right|^{2}+m_{\ell \ell}^{2}}$ and the transverse momentum (invariant mass) of the lepton pair denoted by $\vec{p}_{T}^{\ell \ell}\left(m_{\ell \ell}\right)$. The statistical uncertainty is given by $\sqrt{N_{i, \mathrm{~d}}^{W W}}$, where we combine the contents of the last three bins to ensure gaussianity. Theoretical and systematic uncertainties can be found in Tables 5-7 of the same reference. For the $13 \mathrm{TeV} W^{ \pm} Z$ final state, we make the analysis using the transverse mass distribution in Fig. 4(c) in Ref. [38], which is presented in terms of $W^{ \pm} Z$ signal events in six bins covering all values of $m_{T}^{W Z}$ (last bin containing all data above $600 \mathrm{GeV}$ ) and already background subtracted. From the lower panel of the same figure, we read the statistical, total experimental, and theoretical uncertainties in each bin. ${ }^{1}$

With that information, the procedure to obtain the relevant kinematical distributions predicted in presence of the dimension-six operators is the same followed for our run 1 EWDBD analysis [14]. First we simulate the $W^{+} W^{-}$and $W^{ \pm} Z$ productions using MADGRAPH55 [40] with the UFO files for our effective Lagrangian generated

\footnotetext{
${ }^{1}$ Because the data points give the number of $W Z$ signal events after background subtraction, their statistical errors read from the figure are much larger than $\sqrt{N_{i, \mathrm{~d}}^{W Z}}$.
}

with FeynRules [41,42]. We employ pythia6.4 [43] to perform the parton shower, whereas the fast detector simulation is carried out with DELPHES [44]. In order to account for higher-order corrections and additional detector effects, we simulate SM $W^{+} W^{-}$and $W^{ \pm} Z$ productions in the fiducial region requiring the same cuts and isolation criteria adopted by the corresponding ATLAS studies and normalize our results bin by bin to the experimental collaboration predictions for the kinematical distributions under consideration. Then we apply these correction factors to our simulated $W V$ distributions in the presence of the anomalous couplings.

The statistical confrontation of these predictions with the LHC run 2 data is made by means of a binned loglikelihood function based on the contents of the different bins in the kinematical distribution of each channel. In addition to the statistical errors, we incorporate the systematic and theoretical uncertainties added in quadrature to the uncorrelated statistical error in each bin, assuming some partial correlation among them which we estimate to range between $30 \%$ and $70 \%$ with the information provided. With this, we build the corresponding $\chi_{\text {EWDBD }}^{2}$, which we combine with the EWPD bounds so we have

$\chi_{\text {EWPD+EWDBD }}^{2}\left(f_{B}, f_{W}, f_{W W W}, f_{B W}, f_{\Phi, 1}, f_{\Phi, Q}^{(3)}\right.$,

$$
\left.f_{\Phi, Q}^{(1)}, f_{\Phi, u}^{(1)}, f_{\Phi, d}^{(1)}, f_{\Phi, u d}^{(1)}, f_{\Phi, e}^{(1)}, f_{L L L L}\right) .
$$

For Higgs processes, we use the available data from runs

\begin{tabular}{|c|c|c|}
\hline Source & $\begin{array}{l}\text { Int.Luminosity } \\
\left(\mathrm{fb}^{-1}\right)\end{array}$ & $\begin{array}{l}\text { \# Data } \\
\text { points }\end{array}$ \\
\hline $\begin{array}{l}\text { ATLAS + CMS at } 7 \& 8 \mathrm{TeV} \text { [45] } \\
\text { [Table 8, Fig 27] }\end{array}$ & $5 \& 20$ & $20+1$ \\
\hline ATLAS at $13 \mathrm{TeV}$ [46] [Figs. 6,7] & 79.8 & 9 \\
\hline CMS at $13 \mathrm{TeV}$ [47] [Table 3] & 35.9 & 24 \\
\hline ATLAS at $8 \mathrm{TeV}[48](\gamma Z)$ & 20 & 1 \\
\hline ATLAS at $13 \mathrm{TeV}[49](\gamma Z)$ & 36.1 & 1 \\
\hline ATLAS at $13 \mathrm{TeV}[50]\left(\mu^{+} \mu^{-}\right)$ & 36.1 & 1 \\
\hline
\end{tabular}
1 and 2 from the following sources

that provide us signal strengths or ratios of cross sections and branching ratios. The first three references above contain information on all production mechanisms and almost all decay channels in the figures and tables given in the first column. Moreover, these references also provide 
the correlation matrix among the observables, as well as statistic and systematic errors (for Ref. [47], the correlation matrix can be found in [51]). The fourth and fifth references contain information on the rare decay mode $\gamma Z$, whereas the last one on the $\mu^{+} \mu^{-}$channel.

The statistical comparison of our effective theory predictions with the LHC runs 1 and 2 data is made by means of a $\chi_{\text {Higgs }}^{2}$ function based on these 22 (run 1) +35 (run 2) data points. Adding this to the analysis of EWPD and EWDBD, we construct our global 20 dimensional statistical function:

$\chi_{\mathrm{EWPD}+\mathrm{EWDBD}+\mathrm{Higgs}}^{2}\left(f_{B}, f_{W}, f_{W W W}, f_{B B}, f_{W W}, f_{B W}\right.$,

$$
\begin{aligned}
& f_{G G}, f_{\Phi, 1}, f_{\Phi, 2}, f_{\Phi, Q}^{(3)}, f_{\Phi, Q}^{(1)}, f_{\Phi, u}^{(1)}, f_{\Phi, d}^{(1)}, f_{\Phi, u d}^{(1)}, f_{\Phi, e}^{(1)}, \\
& \left.f_{L L L L}, f_{b}, f_{t}, f_{\tau}, f_{\mu}\right) .
\end{aligned}
$$

\section{RESULTS}

We present in Figs. 1-4 $\Delta \chi^{2}$ profiles (in all cases marginalized with respect to all undisplayed parameters involved in the analysis) for the Wilson coefficients for three sets of analyses which differ in the data samples included:

(i) EWPD: $\Delta \chi_{\text {EWPD }}^{2}$ which constrains the eight coefficients in $\Delta \mathcal{L}_{\text {eff }}^{\text {EWPD }}$, Eq. (2.6). They are given by the green lines in Figs. 1 and 3. This analysis is performed by taking into account only the contributions to the observables that are linear in the anomalous Wilson coefficients; for further detail, see Ref. [26].

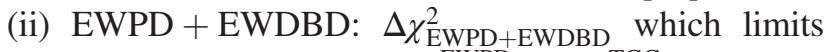
the 12 coefficients in $\Delta \mathcal{L}_{\text {eff }}^{\text {EWPD }}+\Delta \mathcal{L}_{\text {eff }}^{\mathrm{TGC}}$, Eqs. (2.6) and (2.10). The results are depicted in Fig. 2. In the evaluation of the predictions for EWDBD, we have kept the contribution of the Wilson coefficients up to the quadratic order.

(iii) GLOBAL $\equiv$ EWPD + EWDBD + HIGGS: $\Delta \chi_{\text {EWPD+EWDBD+Higgs }}^{2}$ which constrains the 20 coefficients in $\mathcal{L}_{\text {eff }}$ in Eq. (2.15) [Eqs. (2.6)-(2.14)]. They are the red, black, and dashed blue curves in Figs. 1, 3 and 4. In the evaluation of the predictions for EWDBD and the Higgs data, we have kept the contribution of the Wilson coefficients to the physical observables up to the quadratic order.

\section{A. Gauge boson couplings to fermions}

Our results concerning the determination of the Wilson coefficients for the operators involving gauge boson and fermion fields and which directly modify the gauge couplings to fermions are shown in Fig. 1. As is well known, EWPD yields strong bounds on deviations of the SM predictions for the fermion-gauge interactions, and this is quantified in the green curves in the figure. The additional information provided by the inclusion of the LHC data from EWDBD and Higgs observables (now in the larger 20 parameter space) collected at run 1 (and run 2) are shown as the black (red) curves.

In the upper left panel of Fig. 1, we find the $\Delta \chi^{2}$ dependence on $f_{\Phi, e}^{(1)} / \Lambda^{2}$, which is the coefficient of the only operator involving gauge couplings to leptons remaining in the basis after applying the EOM. This operator modifies the $Z$ coupling to right-handed leptons, which were precisely tested at LEP. On the contrary, at the LHC
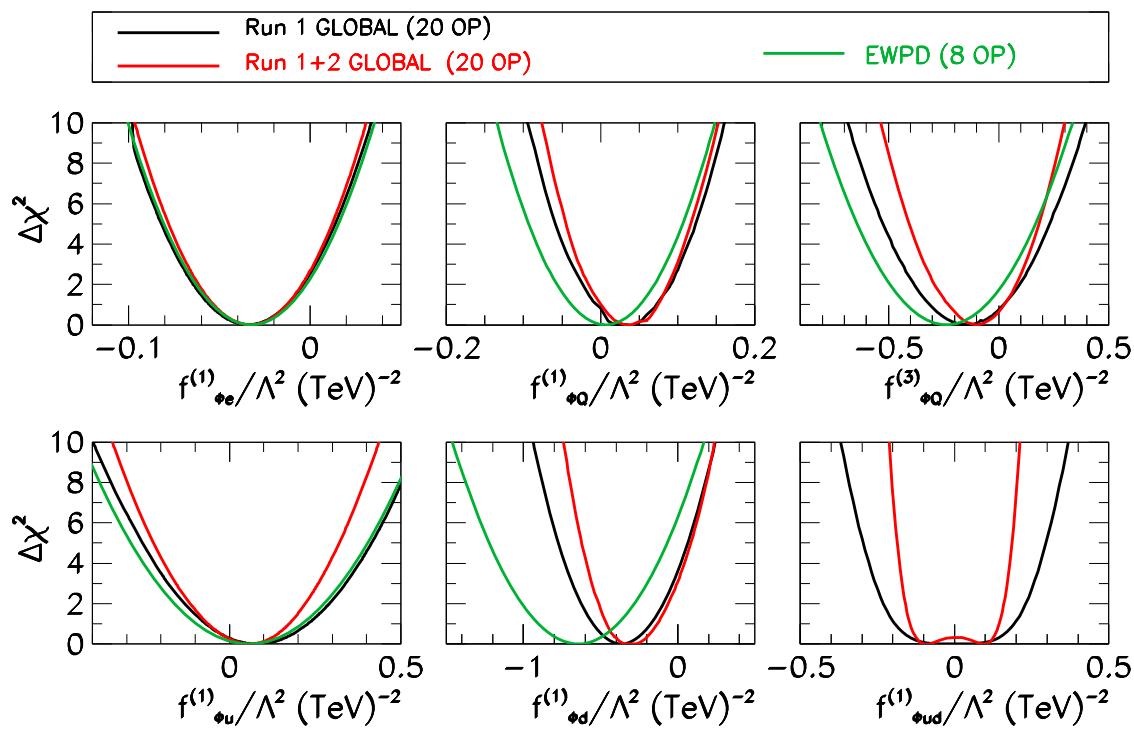

FIG. 1. $\Delta \chi^{2}$ as a function of the fermionic Wilson coefficients $f_{\Phi, e}^{(1)} / \Lambda^{2}, f_{\Phi, Q}^{(1)} / \Lambda^{2}, f_{\Phi, Q}^{(3)} / \Lambda^{2}, f_{\Phi, l}^{(1)} / \Lambda^{2}, f_{\Phi, d}^{(1)} / \Lambda^{2}$, and $f_{\Phi, u d}^{(1)} / \Lambda^{2}$, as indicated in the panels after marginalizing over the remaining fit parameters. The green solid line stands for the fit of the EWPD that constrains only eight of 20 Wilson coefficients in Eq. (2.15). The black (red) solid line represents the 20 parameter fit to the LHC run 1 (and 2) data. 


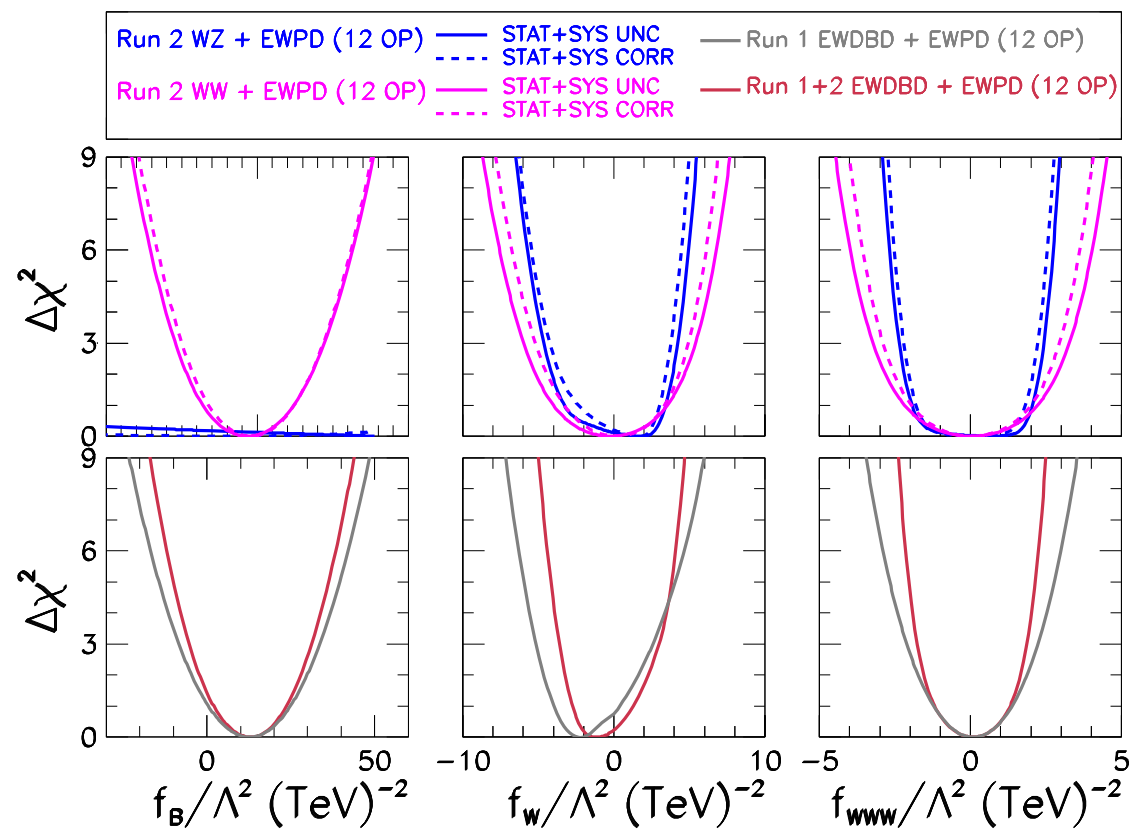

FIG. 2. $\Delta \chi^{2}$ dependence on the $f_{B} / \Lambda^{2}$ (left panel), $f_{W} / \Lambda^{2}$ (central panel) and $f_{W W W} / \Lambda^{2}$ (right panel) parameters after the marginalization over the 11 undisplayed fit parameters for the analysis of LHC EWDBD and EWPD. The upper panels show the results of our analysis of the ATLAS run 2 data on $W Z$ [38] and on $W W$ [39] transverse mass distributions. Full lines (dashed) correspond to assuming zero (total) correlation among the nonstatistical uncertainties; see text for details. The lower panels show the results of the analysis of the EWDBD from run 1 of Ref. [14] in combination with EWPD (black lines) and including also the results from ATLAS $W W$ and $W Z$ production at run 2 (red line).

observables, it enters only via its contribution to the decay rate of the $Z$ boson to leptons in some of the final states considered. Consequently, as seen in the figure, the inclusion of the LHC data does not add any meaningful information about this coefficient.

The central and right upper panels in Fig. 1 display the $\Delta \chi^{2}$ dependence on the coefficients $f_{\Phi, Q}^{(1)} / \Lambda^{2}$ and $f_{\Phi, Q}^{(3)} / \Lambda^{2}$, which correspond to operators modifying the couplings of left-handed quarks to $Z$ and $W$ bosons. On the other hand, the left and central lower panels correspond to the dependence on $f_{\Phi, u}^{(1)} / \Lambda^{2}$ and $f_{\Phi, d}^{(1)} / \Lambda^{2}$, which give corrections to the $u_{R}$ and $d_{R}$ couplings to $Z$, respectively. Comparing the green with the black and red lines, we see that the impact of the inclusion of the LHC results is still minor but not negligible, in particular, for $f_{\Phi d}^{(1)} / \Lambda^{2}$. The EWPD analysis favors nonvanishing value for $f_{\Phi d}^{(1)} / \Lambda^{2}$ at $2 \sigma$, a result driven by the $2.7 \sigma$ discrepancy between the observed $A_{\mathrm{FB}}^{0, b}$ and the $\mathrm{SM}$. On the contrary, no significant discrepancy is observed between the relevant LHC observables, in particular, in EWDBD and the SM predictions. Hence, there is a shift towards zero of $f_{\Phi d}^{(1)} / \Lambda^{2}$ when including the LHC data in the analysis. This slightly smaller tension results also in the reduction of the globally allowed range. This behavior was observed in Ref. [14] for run 1 data, and the inclusion of run 2 results adds in this direction.
Finally, in the right lower panel, we show the $\Delta \chi^{2}$ on the coefficient of $\mathcal{O}_{\Phi, u d}^{(1)}$ operator. This operator induces a righthanded coupling of the $W$ boson to quarks. At the linear level, it does not interfere with the SM and its effect has not been included in the EWPD analysis. The dependence shown in the figure arises from its contribution to the LHC observables which we keep up to the quadratic order (notice that $\Delta \chi^{2}$ as a function of this coupling is symmetric around zero even though its minimum is not exactly at zero). The figure illustrates how including the effect of this operator to that order leads to bounds on its coefficient which are comparable to those of the other operators that modify the coupling of electroweak gauge bosons to quarks and interfere with the SM.

\section{B. Triple anomalous gauge couplings in diboson searches at run 2}

As mentioned in the previous section, for run 2, the number of experimental studies focused on deriving constraints in the size and structure of TGCs is very limited and makes use only of a small fraction of their collected data [37]. However, the ATLAS collaboration has presented results on diboson production in Refs. [38,39], which we make use to test the TGCs; see previous section for details on our construction of the corresponding likelihood functions. 


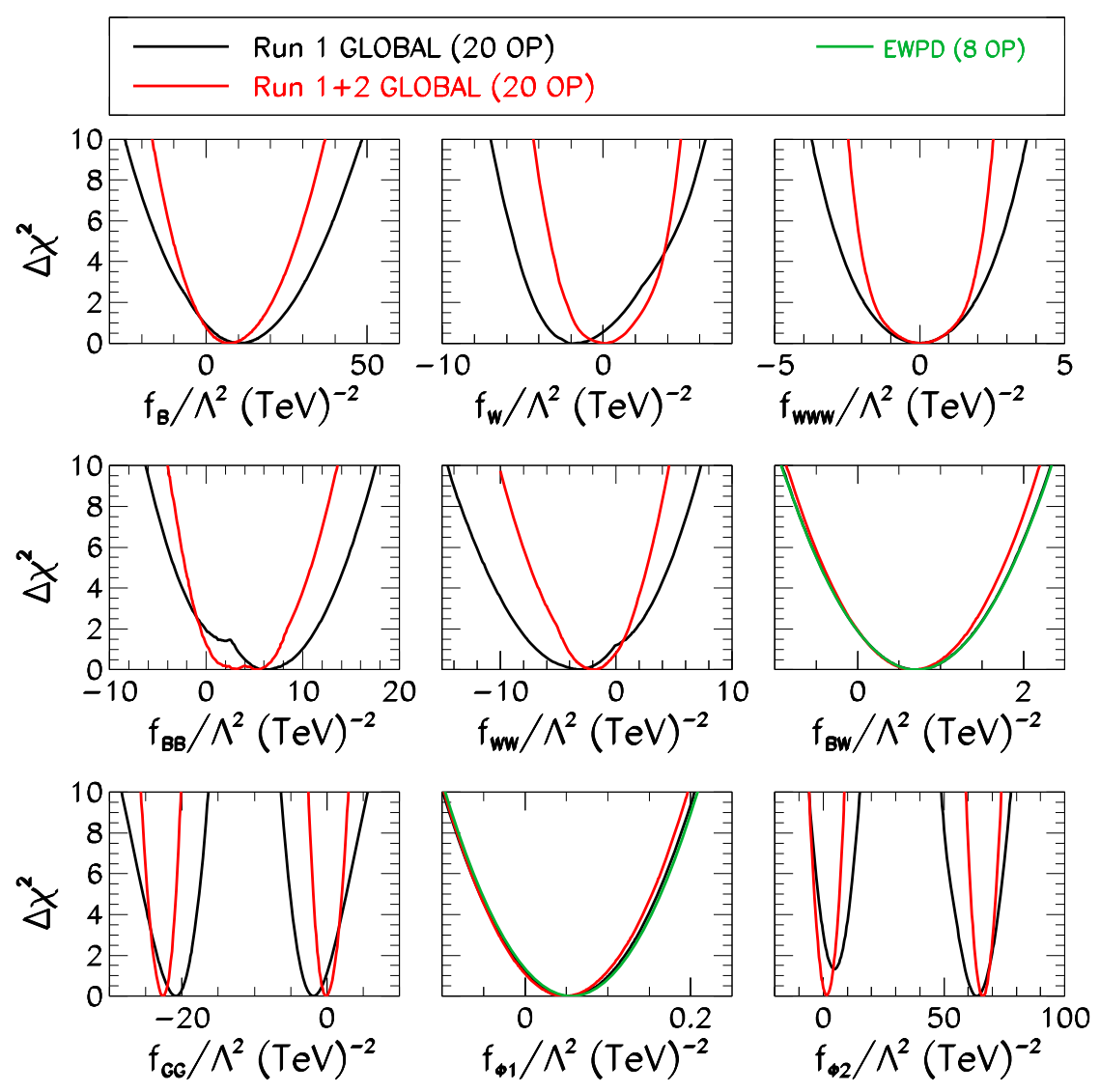

FIG. 3. $\Delta \chi^{2}$ dependence on the bosonic Wilson coefficients $f_{B} / \Lambda^{2}, f_{W} / \Lambda^{2}, f_{W W W} / \Lambda^{2}, f_{B B} / \Lambda^{2}, f_{W W} / \Lambda^{2}, f_{B W} / \Lambda^{2}, f_{G G} / \Lambda^{2}$, $f_{\Phi, 1} / \Lambda^{2}$, and $f_{\Phi, 2} / \Lambda^{2}$ as indicated in each panel. The black (red) line stands for the results of the 20 parameter fit using EWPD, EWDBD, and Higgs data from LHC run 1 (and 2). As before, the green line stands for the fit of only the EWPD.

The results of our analysis of the ATLAS run $2 W W$ and $W Z$ leptonic data $[38,39]$ together with the EWPD in the 12-dimensional parameter space,

$\left\{f_{B}, f_{W}, f_{W W W}, f_{B W}, f_{\Phi, 1}, f_{\Phi, Q}^{(3)}, f_{\Phi, Q}^{(1)}, f_{\Phi, u}^{(1)}, f_{\Phi, d}^{(1)}, f_{\Phi, u d}^{(1)}, f_{\Phi, e}^{(1)}\right\}$

are shown in the upper panels in Fig. 2, where we plot the one-dimensional $\Delta \chi^{2}$ distributions for the Wilson coefficients of the "TGC operators" $\mathcal{O}_{B}, \mathcal{O}_{W}$, and $\mathcal{O}_{W W W}$ after marginalization over the 11 undisplayed coefficients. As expected, the $W Z$ channel gives no constraint on $\mathcal{O}_{B}$, whereas both $W W$ and $W Z$ contribute with similar precision to the determination of $f_{W} / \Lambda^{2}$ and $f_{W W W} / \Lambda^{2}$. To illustrate the possible effect of our assumptions on the correlations of the systematic/theoretical uncertainties (labeled as SYS in the figure) among the different bins, we show the results obtained with full (zero) correlation among those uncertainties in the dashed (solid) lines. As seen in the figure, the effect is small.

In the lower panels, we show the impact of adding the run $2 W W$ and $W Z$ results to the analysis of the run 1 diboson data of Ref. [14], which included data on $W W$ and WZ channels from both ATLAS and CMS collected with $\sim 20 \mathrm{fb}^{-1}$ at each experiment. Altogether we find that the combined ATLAS run 2 diboson data constrain the operator coefficients with precision similar (a bit better indeed) to that of the full run 1 analysis. This is expected from simple statistics of the integrated luminosity and energy scaling. The combination of CMS and ATLAS run 1 data accounts for about $40 \mathrm{fb}^{-1}$ in each $W W$ and $W Z$ channels, which is on the order of the $36 \mathrm{fb}^{-1}$ of ATLAS run 2 data. Moreover, the total cross section for diboson productions is about twice larger at run 2 than at run 1.

\section{Higgs couplings}

Our results concerning the determination of the Wilson coefficients for the operators affecting the interactions of the Higgs field with the gauge bosons and with fermions are shown in Figs. 3 and 4, respectively. In order to perform the most general analysis of the Higgs boson couplings, we use the full dataset that we presented in Sec. III, i.e., EWPD, EWDBD, and Higgs data, including the effect of the 20 operators in Eq. (2.15).

Figure 3 depicts the one-dimensional $\Delta \chi^{2}$ as a function of the Wilson coefficients of the pure bosonic operators in Eq. (2.15) after fitting the EWPD and run 1 (and 2) data on 

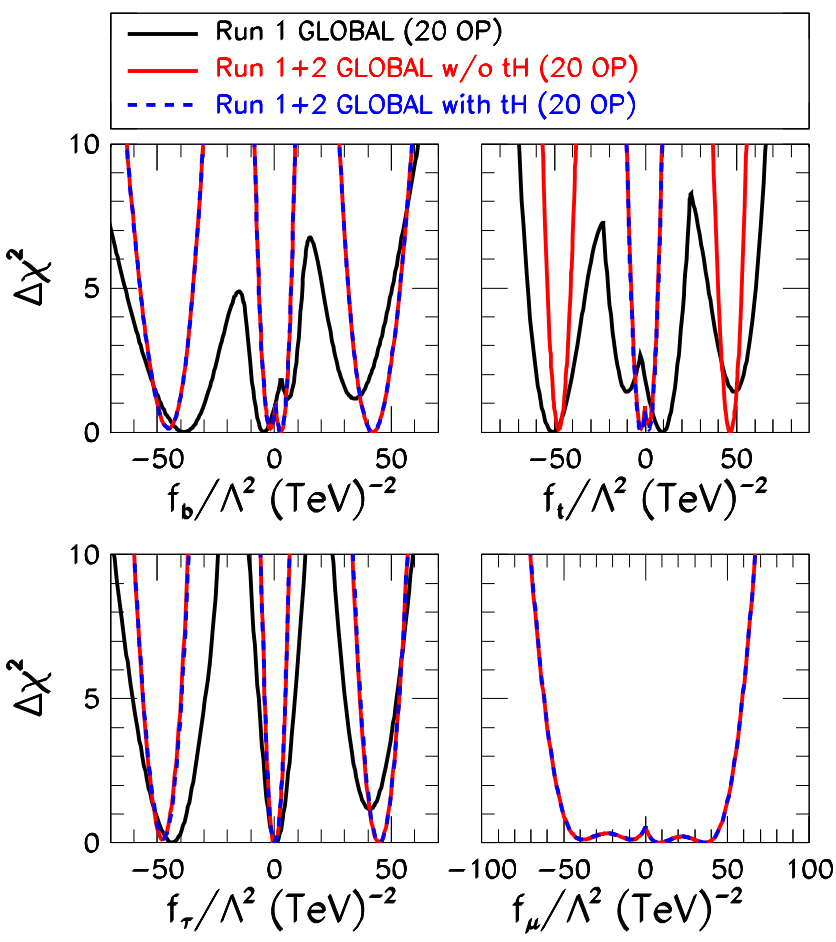

FIG. 4. $\Delta \chi^{2}$ dependence on the fermionic Wilson coefficients $f_{b} / \Lambda^{2}, f_{t} / \Lambda^{2}, f_{\tau} / \Lambda^{2}$, and $f_{\mu} / \Lambda^{2}$ as indicated in each panel. The black (blue) line stands for the results of the 20 parameter fit using EWPD, diboson production, and Higgs data from LHC run 1 (and 2). The red line shows that results obtained using EWPD, diboson production, and Higgs data from LHC runs 1 and 2 without the $t H$ contribution to the Higgs top associate with production cross section (see text for details).

Higgs and diboson productions. As expected, the most stringent constraints are those on the oblique operators $\mathcal{O}_{\Phi, 1}$ and $\mathcal{O}_{B W}$ that come from the EWPD with very little impact of the LHC data [14].

The first row of Fig. 3 contains the $\Delta \chi^{2}$ distributions for the coefficients of the "TGC operators". Of those, only $\mathcal{O}_{B}$ and $\mathcal{O}_{W}$ enter both in TGC and Higgs processes. For completeness, we include here the results of our global analysis on $f_{W W W} / \Lambda^{2}$ also, but we notice that $\mathcal{O}_{W W W}$ does not involve the Higgs field. As we can see, altogether the inclusion of the run 2 data improves the bounds on the coefficients of the three "TGC operators" by a factor $\mathcal{O}(25 \%)$. Also comparing the results for these operators to the second row of Fig. 2, we learn that the inclusion of the Higgs dataset strengthens the bounds on $f_{B} / \Lambda^{2}$ and $f_{W} / \Lambda^{2}$ derived from the EWDBD analysis by $\mathcal{O}(10-20 \%)$ [52].

The operators $\mathcal{O}_{B B}$ and $\mathcal{O}_{W W}$ modify the Higgs decay into two photons with a contribution proportional to $f_{B B} / \Lambda^{2}+f_{W W} / \Lambda^{2}$, therefore, introducing a strong correlation between these operators [6] as the decay rate for this Higgs channel is very well measured. This is illustrated in Fig. 5, where we show the two-dimensional allowed regions from the global analysis for different pairs of
Wilson coefficients after marginalizing over the 18 undisplayed parameters in each panel. In particular, on the left panel, we show the degree of anticorrelation between $f_{B B} / \Lambda^{2}$ and $f_{W W} / \Lambda^{2}$ still present after a combination of run 1 and 2 data. We also see the two quasidegenerate solutions discussed in Eq. (2.19), the lower one containing the SM solution $\left(f_{W W}=f_{B B}=0\right)$ and the upper one with flipped sign of the $H \gamma \gamma$ coupling "displaced" by $\sim 3 \mathrm{TeV}^{-2}$.

The lower row of Fig. 3 contains the results for $f_{G G} / \Lambda^{2}$ and $f_{\Phi, 2} / \Lambda^{2}$, which clearly display the discrete (quasi) degeneracies explained in Sec. II [see discussions around Eqs. (2.18) and (2.16)] associated with the reversion of the sign of the $H g g$ and $H V V(V=Z, W)$ couplings, respectively. Notice that the two solutions for $f_{G G} / \Lambda^{2}$ are completely equivalent in the analysis as this coupling only appears in one vertex. On the other hand, the operator $\mathcal{O}_{\Phi, 2}$ modifies universally all the SM-like Higgs couplings to gauge bosons and fermions. For each of them, there are two degenerate solutions due to the total reversal of the coupling sign, but they would only lie at exactly the same values of $f_{\Phi, 2} / \Lambda^{2}$ if all the couplings were measured to have the same ratio to their SM value. The quasidegeneracy in $f_{\Phi, 2} / \Lambda^{2}$ present in our global analysis originates from the lack of tension between the SM predictions and the data for all processes, so values around $f_{\Phi, 2} / \Lambda^{2} \sim 0$ and $f_{\Phi, 2} / \Lambda^{2} \sim \frac{4}{v^{2}}$ with all other couplings zero can lead to a good global description of the data. As seen in the figure, the addition of run 2 data has contributed in this direction.

We display in Fig. 4 the $\Delta \chi^{2}$ dependence on the Wilson coefficients of the operators generating anomalous Yukawa couplings. Comparing the results obtained using just the LHC run 1 dataset (black curve) with the ones that contains the LHC run 2 data (red curve), we can see that the run 2 data are essential to better constrain these Wilson coefficients. For instance, we can witness the emergence of the three discrete solutions for $f_{b} / \Lambda^{2}$ and $f_{\tau} / \Lambda^{2}$, which originate from Eqs. (2.16) and (2.17) as explained in Sec. II. On the other hand, it is clear from this figure that the data on Higgs decay into muon pairs are still incipient and within the present precision the allowed regions around the three minima merge into a unique allowed range.

We show in this figure the results of the global analysis under two assumptions for the top Higgs associate production in run 2. As described in Ref. [46] both $t t H$ and $t H$ (including $t H W$ and $t H j$ ) contribute to the cross section ratio given in Fig. 6 of that reference. However, with the information provided, it is not possible to determine the relative contribution of $t H$ vs $t t H$ to the reconstructed total cross section ratio. We show the results for two extreme assumptions: a ratio of the $t H$ to $t t H$ contribution as predicted by the model (i.e., exactly same reconstruction efficiency for both subprocesses), shown as the dashed blue lines in the figure, and a negligible small contribution from $t H$ shown in the red line. For consistency, we see that the 

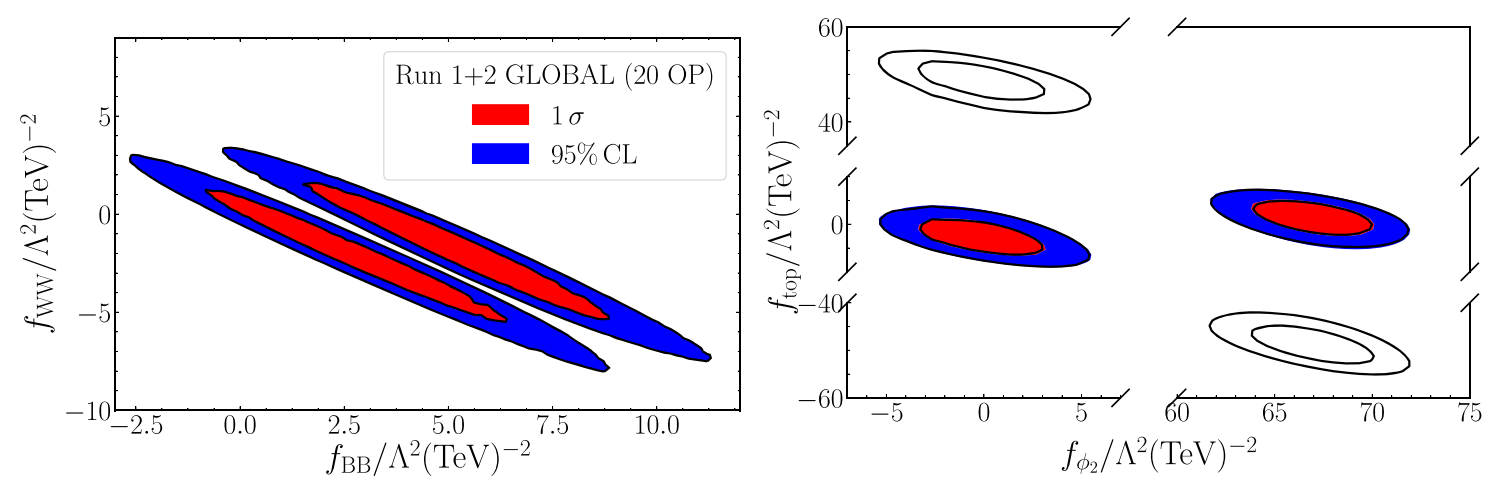

FIG. 5. $1 \sigma$ and 95\% CL (2dof) allowed regions from the global analysis in the planes indicated in the axes. In the right panel, the filled regions are obtained from the global analysis including the $t H$ contribution to the top Higgs associate production data of ATLAS at run 2 , whereas the void regions are the additional solutions allowed when the $t H$ contribution is not included.

results for all nontop Yukawa couplings are exactly the same for the two analyses. For $f_{t} / \Lambda^{2}$, we find that including a "full" $t H$ contribution results into the total breaking of the degeneracies and eliminates solutions other than the ones around $f_{t} / \Lambda^{2}=0$. This can also be seen in the right panel in Fig. 5, where we show the allowed regions in the plane $f_{t} / \Lambda^{2}$ vs $f_{\Phi, 2} / \Lambda^{2}$. The void and colored regions of this panel show the four solutions resulting from Eqs. (2.16) and (2.17) explained in Sec. II, which are quasidegenerated as long as no information on the sign of the $t t H$ coupling is available. On the other hand, the colored regions are the only ones allowed once the information on $t H$ is included in the analysis as described above. This is expected as the $t H$ scattering amplitude receives contributions from the $t t H$ and $V V H$ vertices, therefore, being sensitive to the relative sign of the different diagrams contributing to it [53-55]. In fact, the sign with respect to the SM of the vertices $t t H$ and $V V H$ are the same in the surviving colored regions in Fig. 5. This result clearly illustrates the importance of the measurement of the $t H$ production rate to unambiguously determine the coupling of the Higgs to the top quark. Certainly, the sign of the top Yukawa coupling can be corroborated by other channels; see for instance Refs. [8,56].

\section{Results at linear order}

In order to interpret Eq. (2.1) as a systematic expansion in the large mass parameter $\Lambda$ [3], its contributions to observables at the lowest order $(1 / \Lambda)$ are given the interference between anomalous and SM terms. In principle, if one keeps the quadratic contributions on the Wilson coefficients of dimension-six operators, one should include the interference of dimension-eight operators with the SM as they are on the same order.

Up to this point, we consider the effective Lagrangian Eq. (2.15) as a straw man that we use to probe the standard model couplings assuming that it contains all information on possible new physics. The results obtained are thus physically meaningful as long as no large cancellations between the dimension-six quadratic terms and the (here absent) linear dimension-eight SM interference are expected. Furthermore, this is a pragmatic approach because there are phase space regions where the lowest order systematic expansion fails [13] that is signaled by the cross section being negative! Notwithstanding, the use of the quadratic contributions of the dimension-six operators is justified if the new physics is strongly interacting; see for instance Refs. [57-59]. Indeed, this result is a consequence of naive dimensional analysis [60] and some simple power counting analysis. In any case, Ref. [61] shows that the analysis of the LHC data in terms of dimension-six operators is adequate in almost all realistic weakly coupled scenarios, except in the high-energy tails of distributions.

At this point, we would like to understand the importance of keeping the anomalous quadratic terms in the evaluation of the observables. The result of this exercise certainly depends on the amount of data available. To this end, we redid our 20 parameter fit using only the contributions to the observables at linear order on the Wilson coefficients; our results are depicted in Fig. 6. Comparing the results of the dashed curves in Fig. 6 with the green curves in Fig. 1, we can see that the $\Delta \chi^{2}$ distributions as a function of $\left(f_{B W}, f_{\Phi, 1}, f_{\Phi, Q}^{(3)}, f_{\Phi, Q}^{(1)}, f_{\Phi, u}^{(1)}, f_{\Phi, d}^{(1)}, f_{\Phi, e}^{(1)}, f_{L L L L}\right)$ are very much the same as obtained using only the EWPD (see also Table III). This shows that the contributions to these parameters due to diboson and Higgs data arise mainly from the quadratic terms. Moreover, within the input range of variation of the parameters in the analysis, the operator $\mathcal{O}_{\Phi, u d}$ is not bounded if the observables are evaluated using just the linear terms of their Wilson coefficients, whereas $\mathcal{O}_{W W W}$ is only very weakly constrained. This happens because the dominant contributions of these operators are to helicity amplitudes to which the SM does not contribute [14].

The results in Fig. 6 show that the Higgs data are already precise enough to determine $f_{\Phi, 2} / \Lambda^{2}$ and $f_{G G} / \Lambda^{2}$ in the 

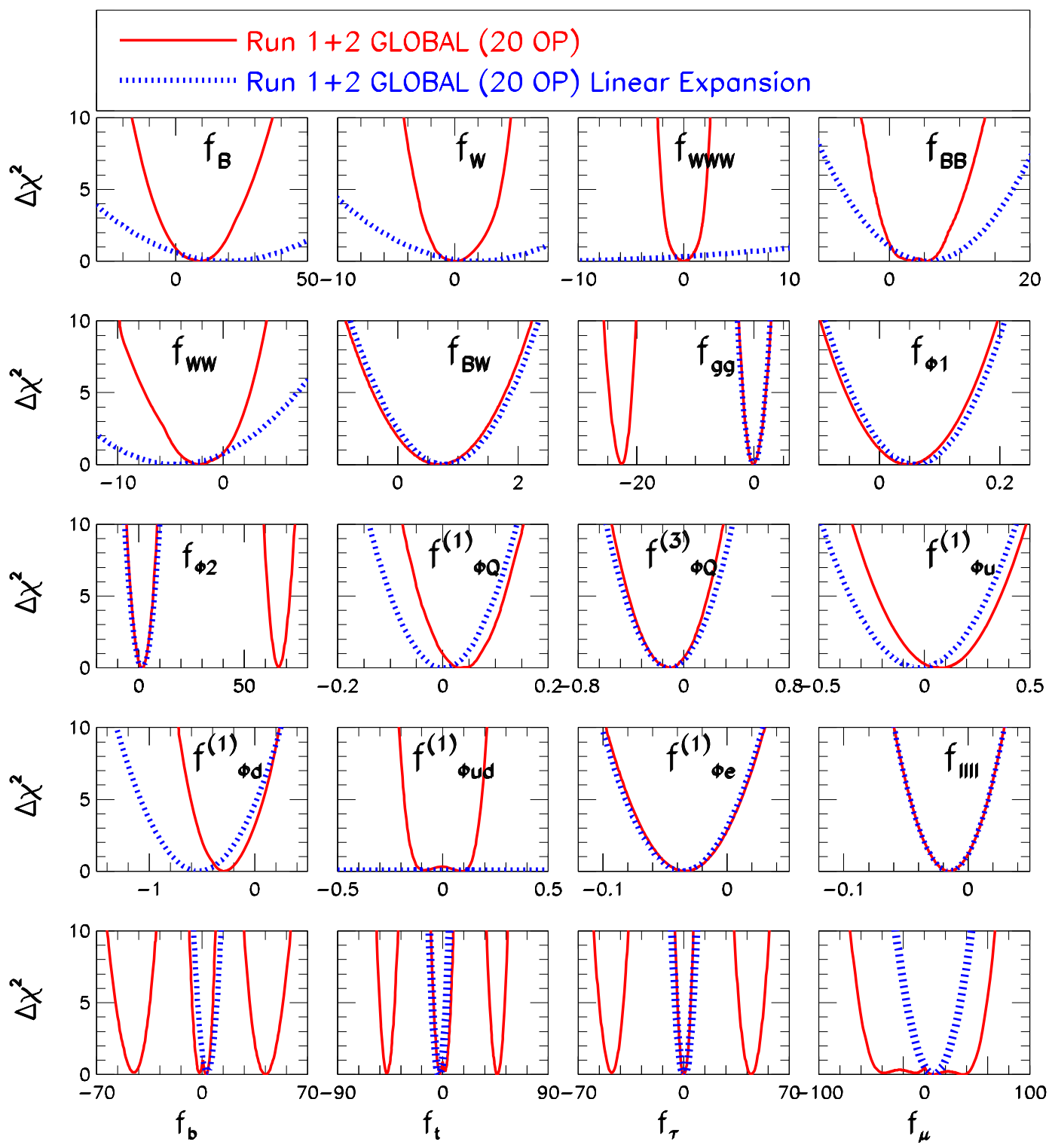

FIG. 6. $\Delta \chi^{2}$ dependence on the bosonic Wilson coefficients $f / \Lambda^{2}$ as indicated in each panel. The results using only the linear terms in the anomalous couplings are indicated by the blue dotted curves. The red solid curves stand for the fits keeping the quadratic contributions of the anomalous couplings to the observables. The full dataset was used in both cases.

linear approximation with the quadratic terms being subdominant. Moreover, as expected, at linear order, there are no degenerate solutions in these couplings, and the allowed regions encompass only the SM. The same applies to the Yukawa-like operators, so the corresponding coefficients $f_{f} / \Lambda^{2}$ have no degenerate solutions. Therefore, the $f_{\mu} / \Lambda^{2}$ seems better determined.

We also find that at present the Higgs data are precise enough to bound the couplings $f_{B B} / \Lambda^{2}$ and $f_{W W} / \Lambda^{2}$ using the linear evaluation of the observables; however, the size of the $95 \% \mathrm{CL}$ allowed area increases by a factor of $\simeq 2$. Similarly, the bounds on $f_{B} / \Lambda^{2}$ and $f_{W} / \Lambda^{2}$ are a factor of $\mathcal{O}(3-4)$ weaker than those obtained when we include the quadratic terms [52]. Nevertheless, this is already very interesting as the diboson production alone does only lead to very mild bounds on these last two couplings when not including the quadratic contributions, which indicates again the relevance of the Higgs observables.

\section{SUMMARY}

In this work, we have performed a comprehensive analysis of the observables related to the electroweak sector, which at present allows for precision tests of the couplings between electroweak gauge bosons and fermions, triple electroweak gauge couplings, and the couplings of the Higgs to fermions and gauge bosons. This includes low-energy electroweak precision measurements 
as well as LHC data on gauge boson pair production and Higgs observables. In total, the GLOBAL analysis of EWPD and EWDBD and Higgs results from LHC run 1 encompasses 64 observables and including runs 1 and 2, 122 observables.

We work in the framework of effective Lagrangians with a linear realization of the electroweak symmetry. At dimension six, and assuming that the new operators do not introduce new tree level sources of flavor violation nor violation of universality of the weak current, the global analysis involves the 20 operators in Eq. (2.15) of which eight contribute to EWPD [Eq. (2.6)], four additional enter in the combination with the LHC EWDBD [Eq. (2.10)], and the 20 operators enter once the Higgs observables are considered.

Altogether the analyses show no statistically significant source of tension with the SM. We find for the SM a $\chi_{\text {SM }}^{2}=$ 118 (71.2) for the 122 (64) observables in the GLOBAL analysis of EWPD and EWDBD and Higgs results from LHC run $1+2$ (only run 1 ). Including the 20 Wilson coefficients in the fit, we find $\chi_{\mathcal{L}_{\text {eff }}}^{2}=91$ (52.3). As a consequence, bounds on the Wilson coefficients can be imposed. The $95 \%$ allowed ranges for the 20 Wilson coefficients (profiled from the global 9-, 12-, or 20-dimensional likelihoods) are listed in Table III. The corresponding allowed 95\% CL ranges for the global analysis with run 1 and run $1+2$ EWDBD and Higgs observables are also graphically displayed in Fig. 7.

In brief, we find:

(i) The coefficients of the eight operators contributing to the EWPD are those better determined, as could be anticipated. The inclusion of the LHC EWDBD and Higgs observables has negligible impact on those operators contributing to the couplings of leptons, $\mathcal{O}_{L L L L}, \mathcal{O}_{\Phi, e}^{(3)}, \mathcal{O}_{\Phi, 1}$, and $\mathcal{O}_{B W}$.

(ii) The impact of the inclusion of the LHC results is still minor but not negligible for the EWPD-bounded operators involving gauge couplings to quarks. In particular, for $f_{\Phi d}^{(1)} / \Lambda^{2}$, under the assumption of generation universality, a nonzero value for this coefficient is favored in the EWPD analysis coming from the $2.7 \sigma$ discrepancy between the observed $A_{\mathrm{FB}}^{0, b}$ and the SM. On the contrary, LHC observables involving this operator are fully consistent with the SM that results in the shift and reduction of its globally allowed range.

(iii) The operator $\mathcal{O}_{\Phi, u d}^{(1)}$ induces right-handed charge current couplings for quarks, and it can only be bound via its quadratic contributions. Including those in the LHC observables, its Wilson coefficient can be bounded with precision comparable to that of the other operators affecting gauge-quark couplings.

TABLE III. $95 \%$ allowed ranges for the Wilson coefficients for the different analysis performed in this work. For $\mathcal{O}_{u \Phi, 33}$, we show in the fifth column the three discrete ranges allowed when no contribution of $t H$ is included in the ATLAS cross section ratio. Including the $t H$ contribution under the assumptions discussed in the text selects the range around zero, which we mark with square brackets.

\begin{tabular}{|c|c|c|c|c|c|}
\hline \multirow[b]{2}{*}{ Operator } & \multicolumn{5}{|c|}{$95 \% \mathrm{CL} f / \Lambda^{2}\left(\mathrm{TeV}^{-2}\right)$} \\
\hline & EWPD & EWPD + EWDBD & GLOBAL Run 1 & GLOBAL Run $1+2$ & $\begin{array}{c}\text { GLOBAL } \\
\text { Run } 1+2 \text { Linear }\end{array}$ \\
\hline $\mathcal{O}_{L L L L}$ & $(-0.043,0.013)$ & $(-0.043,0.013)$ & $(-0.043,0.013)$ & $(-0.043,0.013)$ & $(-0.043,0.013)$ \\
\hline $\mathcal{O}_{\Phi, e}^{(3)}$ & $(-0.075,0.011)$ & $(-0.075,0.007)$ & $(-0.077,0.009)$ & $(-0.075,0.007)$ & $(-0.077,0.005)$ \\
\hline $\mathcal{O}_{\Phi, 1}^{\Phi, c}$ & $(-0.040,0.15)$ & $(-0.040,0.15)$ & $(-0.043,0.15)$ & $(-0.044,0.14)$ & $(-0.034,0.15)$ \\
\hline $\mathcal{O}_{B W}$ & $(-0.32,1.7)$ & $(-0.27,1.7)$ & $(-0.32,1.7)$ & $(-0.30,1.7)$ & $(-0.21,1.8)$ \\
\hline $\mathcal{O}_{\Phi, Q}^{(3)}$ & $(-0.60,0.12)$ & $(-0.45,0.13)$ & $(-0.49,0.11)$ & $(-0.38,0.15)$ & $(-0.41,0.19)$ \\
\hline $\mathcal{O}_{\Phi, O}^{(1)}$ & $(-0.083,0.10)$ & $(-0.034,0.11)$ & $(-0.049,0.11)$ & $(-0.036,0.11)$ & $(-0.089,0.088)$ \\
\hline $\mathcal{O}_{\Phi, d}^{(1)}$ & $(-1.2,-0.13)$ & $(-0.64,-0.007)$ & $(-0.73,0.02)$ & $(-0.56,0.04)$ & $(-1.0,-0.03)$ \\
\hline $\mathcal{O}_{\Phi, u}^{(1)}$ & $(-0.25,0.37)$ & $(-0.17,0.37)$ & $(-0.22,0.38)$ & $(-0.19,0.33)$ & $(-0.32,0.25)$ \\
\hline $\mathcal{O}_{\Phi, u d}^{(1)}$ & $\ldots$ & $(-0.17,0.17)$ & $(-0.29,0.29)$ & $(-0.18,0.18)$ & $\cdots$ \\
\hline $\mathcal{O}_{B}$ & $\cdots$ & $(-7.8,34)$ & $(-12,34)$ & $(-8.3,26)$ & $(-31,70)$ \\
\hline $\mathcal{O}_{W}$ & $\cdots$ & $(-3.9,3.5)$ & $(-5.2,3.5)$ & $(-3.0,3.7)$ & $(-9.5,13)$ \\
\hline $\mathcal{O}_{W W W}$ & $\cdots$ & $(-1.9,2.0)$ & $(-2.6,2.5)$ & $(-1.9,2.0)$ & $(-64,36)$ \\
\hline $\mathcal{O}_{B B}$ & $\cdots$ & $\ldots$ & $(-2.5,13)$ & $(-1.7,10)$ & $(-5.4,16)$ \\
\hline $\mathcal{O}_{W W}$ & $\cdots$ & $\cdots$ & $(-10,3.7)$ & $(-6.7,2.1)$ & $(-15,5.8)$ \\
\hline $\mathcal{O}_{G G}$ & $\cdots$ & $\cdots$ & $(-25,-17) \cup(-4.7,2.1)$ & $(-25,-21) \cup(-1.7 .1,8)$ & $(-1.8,1.7)$ \\
\hline $\mathcal{O}_{\Phi, 2}$ & $\cdots$ & $\cdots$ & $(-1.1,10) \cup(55,72)$ & $(-3.2,6.2) \cup(62,71)$ & $(-3.7,6.9)$ \\
\hline $\mathcal{O}_{d \Phi, 33}$ & $\cdots$ & $\cdots$ & $(-62,-20) \cup(-12,11) \cup(23,45)$ & $(-56,-36) \cup(-6.1,6.7) \cup(33,52)$ & $(-2.2,9.2)$ \\
\hline $\mathcal{O}_{u \Phi, 33}$ & $\cdots$ & $\cdots$ & $(-64,-35) \cup(-19,20) \cup(37,59)$ & $(-53,-42) \cup[-7.4,6.2] \cup(40,52)$ & $(-8.3,2.4)$ \\
\hline $\mathcal{O}_{e \Phi, 33}$ & $\cdots$ & $\cdots$ & $(-59,-31) \cup(-5.8,7.8) \cup(32,50)$ & $(-55,-41) \cup(-3.7,4.3) \cup(37,52)$ & $(-4.8,5.4)$ \\
\hline $\mathcal{O}_{e \Phi, 22}$ & $\cdots$ & $\ldots$ & & $(-50,57)$ & $(-14,31)$ \\
\hline
\end{tabular}




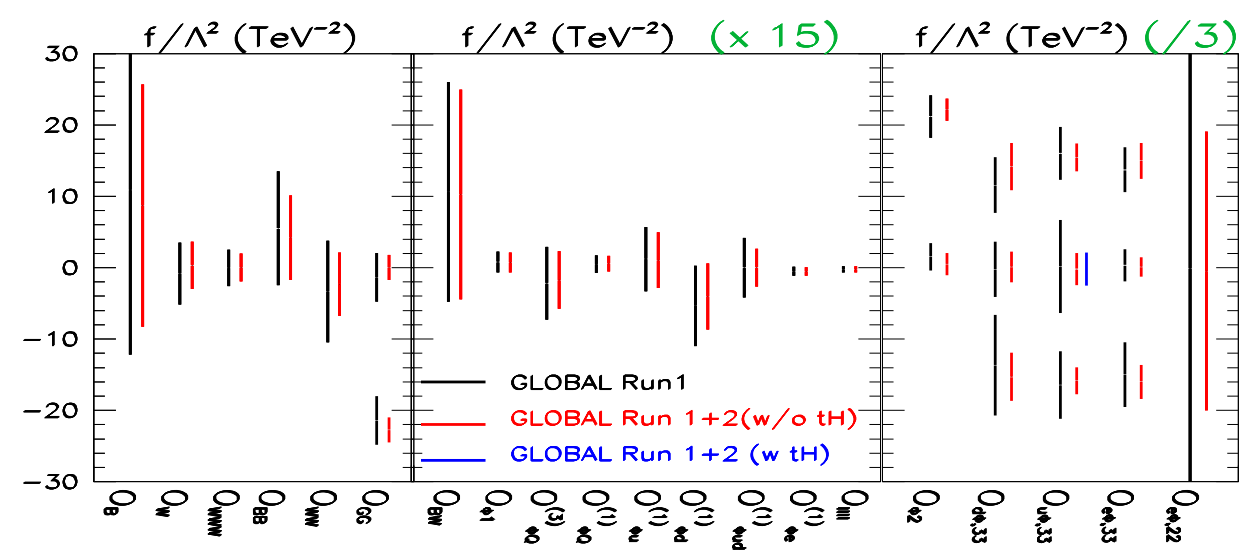

FIG. 7. Allowed 95\% CL ranges for each of the Wilson coefficients from the 20 parameter global analysis. We show results from the global EWPD + LHC analysis including run 1 EWDB and Higgs observables only (black bars) and including both runs 1 and 2 EWDB and Higgs observables (red line). For the top Yukawa-like operator $\mathcal{O}_{u \Phi, 33}$, we show as a blue bar the allowed range when considering the contribution from $t H j$ and $t H W$ in the to the top Higgs associate production. For the graphical display, the ranges in the central (right) panels are multiplied (divide) by a factor 30 (3) as indicated on the top.

(iv) We have performed a novel analysis of the run 2 ATLAS $36.1 \mathrm{fb}^{-1}$ data on transverse mass distribution of $W^{+} W^{-}$and $W^{ \pm} Z$ in the leptonic channel $[38,39]$, which allows for further tests of the TGCs. The results are shown in Fig. 2. We find that the combined ATLAS run 2 diboson data constrains the operator coefficients with precision similar (a bit better indeed) to that of the full run 1 analysis.

(v) Inclusion of the run 2 results in the global analysis results in a reduction of the allowed range for the coefficients of the bosonic operators $\mathcal{O}_{B}, \mathcal{O}_{W}$, $\mathcal{O}_{W W W}, \mathcal{O}_{B B}$, and $\mathcal{O}_{W W}$ by $20-30 \%$.

(vi) The allowed values for $f_{G G} / \Lambda^{2}$ and $f_{\Phi, 2} / \Lambda^{2}$ present two discrete ranges originated by the degeneracy (it is a quasidegeneracy for $f_{\Phi, 2}$ ) associated with the reverse of the sign of the $H g g$ [Eq. (2.18)] and $H X X$ $(X=f, V)$ [Eqs. (2.16) and (2.17)], respectively. Barring that these are degenerate solutions, these are the best determined coefficients for operators not contributing to EWPD.

(vii) The allowed values for the coefficients for the Yukawa-like operators $\mathcal{O}_{d \Phi, 33}$ and $\mathcal{O}_{e \Phi, 33}\left(f_{b}\right.$, and $f_{\tau}$ ) have a two folded degeneracy associated with the reverse of the sign of the corresponding $\mathrm{Hff}$ coupling [see Eq. (2.17)] in combination with the reverse of the sign of the $H V V$ coupling. This results in the three discrete allowed ranges in Table III. For the $\mathcal{O}_{e \Phi, 22}$ coefficient, $f_{\mu}$, the data are not precise enough to resolve the three solutions.

(viii) For $\mathcal{O}_{u \Phi, 33}$, the inclusion of the incipient $t H$ data can break those degeneracies on $f_{t}$; this is in the determination of the top Higgs coupling.

(ix) The last column in Table III shows the allowed ranges when only the terms linear in the Wilson coefficients are kept in the observables. For those operators constrained by EWPD, the bounds are just that of the EWPD, but at LHC, they are mainly constrained by its quadratic contribution. For the operators without degenerate solutions, the bounds become weaker but are still within the same order or magnitude. Exceptions are $\mathcal{O}_{\Phi, u d}^{(1)}$ and $\mathcal{O}_{W W W}$, which become very weakly bounded as their dominant contributions at LHC are to helicity amplitudes which do not interfere with the SM ones. Keeping only the linear contribution to the observables does not allow for the degenerated solutions associated with the sign flip of the Higgs couplings. Consequently, $f_{G G} / \Lambda^{2}, f_{\Phi, 2} / \Lambda^{2}, f_{B} / \Lambda^{2}, f_{t} / \Lambda^{2}, f_{\tau} / \Lambda^{2}$, and $f_{\mu} / \Lambda^{2}$ appear to be better constrained.

(x) Our results show the importance of the Higgs data to constrain the TGC operators $\mathcal{O}_{B}$ and $\mathcal{O}_{W}$ when the LHC observables are evaluated using only the linear terms in the anomalous couplings. This extends the previous results in Refs. [52,62].

Altogether we find that the increased integrated luminosity gathered at $13 \mathrm{TeV}$ allows us to obtain more stringent bounds on a larger set of anomalous interactions and to perform new tests of the SM. We look forward for the release of the complete dataset accumulated at run 2 .

\section{ACKNOWLEDGMENTS}

We thank Tyler Corbett for discussions. We are indebted to Juan Gonzalez-Fraile for his help with comparison with previous analysis. M. C. G.-G. and N. R. A. thank the group at Universidade de Sao Paulo for their hospitality. O. J. P. E. wants to thank the group at the Universitat de Barcelona for the hospitality during the final stages of this work. This work is supported in part by Conselho Nacional de Desenvolvimento Científico e Tecnológico (CNPq) and by Fundação de Amparo à Pesquisa do Estado de São Paulo (FAPESP) Grants No. 2012/10095-7 and No. 2017/06109-5, 
by USA-NSF Grant No. PHY-1620628, by EU Networks FP10 ITN ELUSIVES (H2020-MSCA-ITN-2015-674896) and INVISIBLES-PLUS (H2020-MSCA-RISE-2015690575), by MINECO Grant No. FPA2016-76005-C2-1-P and by Maria de Maeztu program Grant No. MDM-20140367 of ICCUB. A. A. thanks Conselho Nacional de Desenvolvimento Científico $(\mathrm{CNPq})$ for its financial support, Grant No. 307265/2017-0.
[1] G. Aad et al. (ATLAS Collaboration), Phys. Lett. B 716, 1 (2012).

[2] S. Chatrchyan et al. (CMS Collaboration), Phys. Lett. B 716, 30 (2012).

[3] S. Weinberg, Physica (Amsterdam) 96A, 327 (1979).

[4] H. Georgi, Weak Interactions and Modern Particle Theory (Benjamin/cummings, 1984), ISBN 9780805331639.

[5] J. F. Donoghue, E. Golowich, and B. R. Holstein, Cambridge Monogr. Part. Phys., Nucl. Phys., Cosmol. 2, 1 (1992); 35, 1 (2014).

[6] T. Corbett, O. J. P. Éboli, J. González-Fraile, and M. C. González-Garcia, Phys. Rev. D 86, 075013 (2012).

[7] T. Corbett, O. J. P. Éboli, J. González-Fraile, and M. C. González-Garcia, Phys. Rev. D 87, 015022 (2013).

[8] T. Corbett, O. J. P. Éboli, D. Goncalves, J. González-Fraile, T. Plehn, and M. Rauch, J. High Energy Phys. 08 (2015) 156.

[9] A. Butter, O. J. P. Éboli, J. Gonzalez-Fraile, M. C. GonzalezGarcia, T. Plehn, and M. Rauch, J. High Energy Phys. 07 (2016) 152.

[10] J. Ellis, C. W. Murphy, V. Sanz, and T. You, J. High Energy Phys. 06 (2018) 146.

[11] C. Grojean, M. Montull, and M. Riembau, arXiv: 1810.05149.

[12] Z. Zhang, Phys. Rev. Lett. 118, 011803 (2017).

[13] J. Baglio, S. Dawson, and I. M. Lewis, Phys. Rev. D 96, 073003 (2017).

[14] A. Alves, N. Rosa-Agostinho, O. J. P. Éboli, and M. C. Gonzalez-Garcia, Phys. Rev. D 98, 013006 (2018).

[15] S. Weinberg, Phys. Rev. Lett. 43, 1566 (1979).

[16] B. Grzadkowski, M. Iskrzynski, M. Misiak, and J. Rosiek, J. High Energy Phys. 10 (2010) 085.

[17] H. D. Politzer, Nucl. Phys. B172, 349 (1980).

[18] H. Georgi, Nucl. Phys. B361, 339 (1991).

[19] C. Arzt, Phys. Lett. B 342, 189 (1995).

[20] H. Simma, Z. Phys. C 61, 67 (1994).

[21] K. Hagiwara, S. Ishihara, R. Szalapski, and D. Zeppenfeld, Phys. Rev. D 48, 2182 (1993).

[22] K. Hagiwara, T. Hatsukano, S. Ishihara, and R. Szalapski, Nucl. Phys. B496, 66 (1997).

[23] S. Schael et al. (SLD Electroweak Group, SLD Heavy Flavour Group, LEP Electroweak Working Group, DELPHI, ALEPH, SLD, OPAL, and L3 Collaborations), Phys. Rep. 427, 257 (2006).

[24] A. De Rujula, M. B. Gavela, P. Hernandez, and E. Masso, Nucl. Phys. B384, 3 (1992).

[25] J. Elias-Miro, J. R. Espinosa, E. Masso, and A. Pomarol, J. High Energy Phys. 11 (2013) 066.
[26] T. Corbett, O. J. P. Éboli, and M. C. Gonzalez-Garcia, Phys. Rev. D 96, 035006 (2017).

[27] T. Corbett, O. J. P. Éboli, and M. C. Gonzalez-Garcia, Phys. Rev. D 91, 035014 (2015).

[28] C. Patrignani et al. (Particle Data Group), Chin. Phys. C 40, 100001 (2016).

[29] L.E. W. Group (Tevatron Electroweak Working Group, SLD Electroweak and Heavy Flavour Groups, LEP Electroweak Working Group, CDF, DELPHI, ALEPH, SLD, OPAL, D0, and L3 Collaborations), arXiv:1012.2367.

[30] M. Ciuchini, E. Franco, S. Mishima, M. Pierini, L. Reina, and L. Silvestrini, in Proceedings of the International Conference on High Energy Physics 2014 (ICHEP 2014), Valencia, Spain, 2014 (Elsevier, 2014); Nucl. Part. Phys. Proc. 273275, 2219 (2016).

[31] LEP, ALEPH, DELPHI, L3, and OPAL Collaborations and the LEP TGC Working Group, A Combination of Preliminary Results on Gauge Boson Couplings Measured by the LEP Experiments, http://lepewwg.web.cern.ch/ LEPEWWG/lepww/tgc, lEPEWWG/TGC/2002-02.

[32] G. Aad et al. (ATLAS Collaboration), J. High Energy Phys. 09 (2016) 029.

[33] V. Khachatryan et al. (CMS Collaboration), Eur. Phys. J. C 76, 401 (2016).

[34] G. Aad et al. (ATLAS Collaboration), Phys. Rev. D 93, 092004 (2016).

[35] V. Khachatryan et al. (CMS Collaboration), Eur. Phys. J. C 77, 236 (2017).

[36] K. Hagiwara, R. D. Peccei, D. Zeppenfeld, and K. Hikasa, Nucl. Phys. B282, 253 (1987).

[37] G. Aad et al. (ATLAS Collaboration), Report No. ATLASCONF-2016-043, https://cds.cern.ch/record/2206093.

[38] G. Aad et al. (ATLAS Collaboration), Report No. ATLASCONF-2018-034, https://cds.cern.ch/record/2630187.

[39] M. Aaboud et al. (ATLAS Collaboration), Eur. Phys. J. C 78, 24 (2018).

[40] J. Alwall, R. Frederix, S. Frixione, V. Hirschi, F. Maltoni, O. Mattelaer, H. S. Shao, T. Stelzer, P. Torrielli, and M. Zaro, J. High Energy Phys. 07 (2014) 079.

[41] N. D. Christensen and C. Duhr, Comput. Phys. Commun. 180, 1614 (2009).

[42] A. Alloul, N. D. Christensen, C. Degrande, C. Duhr, and B. Fuks, Comput. Phys. Commun. 185, 2250 (2014).

[43] T. Sjostrand, S. Mrenna, and P. Z. Skands, J. High Energy Phys. 05 (2006) 026.

[44] J. de Favereau, C. Delaere, P. Demin, A. Giammanco, V. Lemaitre, A. Mertens, and M. Selvaggi (DELPHES 3 Collaboration), J. High Energy Phys. 02 (2014) 057. 
[45] G. Aad et al. (ATLAS and CMS Collaborations), J. High Energy Phys. 08 (2016) 045.

[46] G. Aad et al. (ATLAS Collaboration), Report No. ATLASCONF-2018-031, https://cds.cern.ch/record/2629412.

[47] A. M. Sirunyan et al. (CMS Collaboration), arXiv: 1809.10733 [Eur. Phys. J. (to be published)].

[48] G. Aad et al. (ATLAS Collaboration), Eur. Phys. J. C 76, 6 (2016).

[49] M. Aaboud et al. (ATLAS Collaboration), J. High Energy Phys. 10 (2017) 112.

[50] G. Aad et al. (ATLAS Collaboration), Report No. ATLASCONF-2018-026, http://cds.cern.ch/record/2628763.

[51] A. M. Sirunyan et al. (CMS Collaboration), arXiv: 1809.10733 [Eur. Phys. J. (to be published)].

[52] T. Corbett, O. J. P. Éboli, J. González-Fraile, and M. C. González-Garcia, Phys. Rev. Lett. 111, 011801 (2013).

[53] V. Barger, M. McCaskey, and G. Shaughnessy, Phys. Rev. D 81, 034020 (2010).
[54] S. Biswas, E. Gabrielli, F. Margaroli, and B. Mele, J. High Energy Phys. 07 (2013) 073.

[55] J. Chang, K. Cheung, J. S. Lee, and C.-T. Lu, J. High Energy Phys. 05 (2014) 062.

[56] D. Goncalves, F. Krauss, S. Kuttimalai, and P. Maierhfer, Phys. Rev. D 92, 073006 (2015).

[57] A. Biektter, A. Knochel, M. Krmer, D. Liu, and F. Riva, Phys. Rev. D 91, 055029 (2015).

[58] R. Contino, A. Falkowski, F. Goertz, C. Grojean, and F. Riva, J. High Energy Phys. 07 (2016) 144.

[59] A. Falkowski, M. Gonzalez-Alonso, A. Greljo, D. Marzocca, and M. Son, J. High Energy Phys. 02 (2017) 115.

[60] A. Manohar and H. Georgi, Nucl. Phys. B234, 189 (1984).

[61] J. Brehmer, A. Freitas, D. Lopez-Val, and T. Plehn, Phys. Rev. D 93, 075014 (2016).

[62] F. de Campos, M. C. Gonzalez-Garcia, and S. F. Novaes, Phys. Rev. Lett. 79, 5210 (1997). 